\title{
Hesitant Probabilistic Fuzzy Information Aggregation Using Einstein Operations
}

\author{
Jin Han Park ${ }^{1, *}$, Yu Kyoung Park ${ }^{1}$ and Mi Jung Son ${ }^{2, *}$ \\ 1 Department of Applied Mathematics, Pukyong National University, Busan 608-737, Korea; \\ ykpark87@pknu.ac.kr \\ 2 Department of Mathematics, Korea Maritime University, Busan 606-791, Korea \\ * Correspondence: jihpark@pknu.ac.kr (J.H.P.), mjson72@korea.com (M.J.S.); \\ Tel.: +82-51-629-5530 (J.H.P.); +82-10-4542-0023 (M.J.S.)
}

Received: 8 August 2018; Accepted: 3 September 2018; Published: 4 September 2018

\begin{abstract}
In this paper, a hesitant probabilistic fuzzy multiple attribute group decision making is studied. First, some Einstein operations on hesitant probability fuzzy elements such as the Einstein sum, Einstein product, and Einstein scalar multiplication are presented and their properties are discussed. Then, several hesitant probabilistic fuzzy Einstein aggregation operators, including the hesitant probabilistic fuzzy Einstein weighted averaging operator and the hesitant probabilistic fuzzy Einstein weighted geometric operator and so on, are introduced. Moreover, some desirable properties and special cases are investigated. It is shown that some existing hesitant fuzzy aggregation operators and hesitant probabilistic fuzzy aggregation operators are special cases of the proposed operators. Further, based on the proposed operators, a new approach of hesitant probabilistic fuzzy multiple attribute decision making is developed. Finally, a practical example is provided to illustrate the developed approach.
\end{abstract}

Keywords: hesitant probabilistic fuzzy element (HPFE); Einstein operations; hesitant probabilistic fuzzy Einstein aggregation operators; multiple attribute decision making (MADM).

\section{Introduction}

Decision making problems typically consist of finding the most desirable alternative(s) out of a given set of alternatives. So far, there are applications of decision making into different disciplines, such as railroad container terminal selection, pharmaceutical supplying, hospital service quality, and so on [1-3]. Due to the increasing ambiguity and complexity of the socio-economic environment, it is difficult to obtain accurate and sufficient data for practical decision making. Therefore, uncertainty data needs to be addressed in the actual decision making process, and several other methodologies and theories have been proposed. Among them, the fuzzy set theory [4] is excellent and has been widely used in many areas of real life [5-8]. Since Zadeh [4] introduced the fuzzy set (FS) in 1965, many researchers have developed extended forms of FS, such as the intuitive fuzzy set (IFS) [9], the type-2 fuzzy set [10], the type- $n$ fuzzy set [10], the fuzzy multiset [11] and the fuzzy hesitant set (HFS) [12]. Among these, the HFS was broadly applied to the practical decision making process. In fact, the HFS is widely used in decision making problems with the aim of resolving the difficulty of explaining hesitation in the actual assessment. The main reason is that experts may face situations in which people are hesitant to provide their preferences in the decision making process by allowing them to prefer several possible values between 0 and 1. Torra [12] introduced some basic operations of HFSs. Xia and Xu [13] defined the hesitant fuzzy element (HFE), which is the basic component of the HFS, and proposed and investigated the score function and comparison law of HFEs as the basis for its calculation and application. Li et al. [14] and Meng and Chen [15] proposed various distance measures and some correlation coefficients for HFSs. They also investigated applications based on the 
distance measures and correlation coefficients. Over the past decade, there many researchers [16-23] have studied the aggregation operators, one of the core issues of HFSs. Thus, many researchers have worked hard to develop the HFS theory and have helped to develop it in uncertain decision making problems [24-26].

However, there is one obvious weakness in the current approaches; namely, each of the possible values in the HFE provided by the experts has the same weight. To overcome this weakness, $\mathrm{Xu}$ and Zhou [27] proposed the hesitant probabilistic fuzzy set (HPFS) and hesitant probabilistic fuzzy element (HPFE) developed by introducing probabilities to HFS and HFE respectively. For example, experts evaluate a house's "comfort" using an HFE $(0.3,0.4,0.5)$ because they hesitate to evaluate it. However, they believe that 0.4 is appropriate and 0.3 is less appropriate than the other values in the HFE. Therefore, although the HFE $(0.3,0.4,0.5)$ cannot fully represent the evaluation, the HPFE $(0.3|0.2,0.4| 0.5,0.5 \mid 0.3)$ can present this issue vividly and is more convenient than HFE. Consequently, the HPFS can overcome the defect of HFS to great extent, so it can remain the experts' evaluation information and describe their preferences better. In Ref. [27], the HPFE was combined with weighted operators to develop basic weighted operators, such as hesitant probabilistic fuzzy weighted average/geometric (HPFWA or HPFWG) operators and the hesitant probabilistic fuzzy ordered weighted averaging/geometric (HPFOWA or HPFOWG) operators. Based on the perspective of the aggregation operators, they established the consensus among decision makers in group decision making. Zhang and $\mathrm{Wu}$ [28] investigated some operations of HPFE and applied them to multicriteria decision making (MCDM). In another way, some scholars recently tried to solve the problem of HFSs. Bedregal et al. [29] tried to use fuzzy multisets to improve the HFSs. This method has been worked out to some extent. Wang and Li [30] proposed the picture hesitant fuzzy set to express the uncertainty and complexity of experts' opinions and applied them to solve diverse situations during MCDM processes. Interval-valued HFSs have been used in the applications of group decision making in [31]. Multiple attribute decision making (MADM) using the trapezoidal valued HFSs is discussed in [32]. Yu [33] gave the concept of triangular hesitant fuzzy sets and used it for the solution of decision making problems. Mahmood et al. [34] introduced the cubic hesitant fuzzy set and applied it to MCDM.

The study on aggregation operators to fuse hesitant probabilistic information is one of the core issues in HPFS theory. The all aggregation operators introduced previously, such as the HPFWA, HPFWG, HPFOWA, and HPFOWG operators, are based on the algebraic product and algebraic sum of HPFEs, which are a pair of the special dual $t$-norm and $t$-conorm [35]. Although the algebraic product and algebraic sum are the basic algebraic operations of HPFEs, they are not the only ones. The Einstein product and Einstein sum are good alternatives to the algebraic product and algebraic sum for structuring aggregation operators, respectively, and they have been used to aggregate the intuitionistic fuzzy values or the HFEs by many researchers [21-23,36-38]. However, it seems that in the literature, there has been little investigation on aggregation techniques using the Einstein operations to aggregate hesitant probabilistic fuzzy information. Thus, it is meaningful to research the hesitant probabilistic fuzzy information aggregation methods based on the Einstein operations. In this paper, motivated by the works of $\mathrm{Xu}$ and Zhou [27] and Yu [21], we propose the hesitant probabilistic fuzzy Einstein weighted aggregation operators with the help of Einstein operations, and apply them to MADM under a hesitant probabilistic fuzzy environment. To do this, the remainder of this paper is organized as follows: The following section recalls briefly some basic concepts and notions related to the HPFSs and HPFEs. In Section 3, based on the hesitant probabilistic fuzzy weighted aggregation operator and the Einstein operations, we propose the hesitant probabilistic fuzzy Einstein weighted aggregation operators including the hesitant probabilistic fuzzy Einstein weighted averaging/geometric (HPFEWA or HPFEWG) operators and the hesitant probabilistic fuzzy Einstein ordered weighted averaging/geometric (HPFEOWA or HPFEOWG) operators. Section 4 develops an approach to MADM with hesitant probabilistic fuzzy information based on the proposed operators. An example is given to demonstrate the practicality and effectiveness of the proposed approach in Section 4 . Section 5 gives some concluding remarks. 


\section{Hesitant Fuzzy Information with Probabilities}

\subsection{HPFS and HPFE}

The HPFS and HPFE represent hesitant fuzzy information with the following probabilities.

Definition 1. [27] Let $R$ be a fixed set, then an HPFS on $R$ is expressed by a mathematical symbol:

$$
H_{P}=\left\{\bar{h}\left(\gamma_{i} \mid p_{i}\right) \mid \gamma_{i}, p_{i}\right\}
$$

where $\bar{h}\left(\gamma_{i} \mid p_{i}\right)$ is a set of some elements $\left(\gamma_{i} \mid p_{i}\right)$ denoting the hesitant fuzzy information with probabilities to the set $H_{P}, \gamma_{i} \in R, 0 \leq \gamma_{i} \leq 1, i=1,2, \ldots$,\#商, where $\# \bar{h}$ is the number of possible elements in $\bar{h}\left(\gamma_{i} \mid p_{i}\right)$, $p_{i} \in[0,1]$ is the hesitant probability of $\gamma_{i}$, and $\sum_{i=1}^{\# \bar{h}} p_{i}=1$.

For convenience, $\mathrm{Xu}$ and Zhou [27] called $\bar{h}\left(\gamma_{i} \mid p_{i}\right)$ a HPFE, and $H_{P}$ the set of HPFSs. In addition, they gave the following score function, deviation function, and comparison law to compare different HPFEs.

Definition 2. [27] Let $\bar{h}\left(\gamma_{i} \mid p_{i}\right)(i=1,2, \ldots, \# \bar{h})$ be a HPFE, then

(1) $s(\bar{h})=\sum_{i=1}^{\# \bar{h}} \gamma_{i} p_{i}$ is called the score function of $\bar{h}\left(\gamma_{i} \mid p_{i}\right)$, where $\# \bar{h}$ is the number of possible elements in $\bar{h}\left(\gamma_{i} \mid p_{i}\right)$;

(2) $d(\bar{h})=\sum_{i=1}^{\# \bar{h}}\left(\gamma_{i}-s(\bar{h})\right)^{2} p_{i}$ is called the deviation function of $\bar{h}\left(\gamma_{i} \mid p_{i}\right)$, where $s(\bar{h})=\sum_{i=1}^{\# \bar{h}} \gamma_{i} p_{i}$ is the score function of $\bar{h}\left(\gamma_{i} \mid p_{i}\right)$, and $\# \bar{h}$ is the number of possible elements in $\bar{h}\left(\gamma_{i} \mid p_{i}\right)$.

If all probabilities are equal, i.e., $p_{1}=p_{2}=\cdots=p_{\# \bar{h}}$, then the HPFE is reduced to the HFE. So, in this case, the score function of the HPFE is consistent with that of the HFE.

Definition 3. [27] Let $\bar{h}_{1}\left(\gamma_{i} \mid p_{i}\right)\left(i=1,2, \ldots, \# \bar{h}_{1}\right)$ and $\bar{h}_{2}\left(\gamma_{j} \mid p_{j}\right)\left(j=1,2, \ldots, \# \bar{h}_{2}\right)$ be two HPFEs, $s\left(\bar{h}_{1}\right)$ and $s\left(\bar{h}_{2}\right)$ are the score functions of $\bar{h}_{1}$ and $\bar{h}_{2}$, respectively, and $d\left(\bar{h}_{1}\right)$ and $d\left(\bar{h}_{2}\right)$ are the deviation functions of $\bar{h}_{1}$ and $\bar{h}_{2}$, respectively, then

(1) If $s\left(\bar{h}_{1}\right)<s\left(\bar{h}_{2}\right)$, then $\bar{h}_{1}$ is smaller than $\bar{h}_{2}$ which is denoted by $\bar{h}_{1}<\bar{h}_{2}$;

(2) If $s\left(\bar{h}_{1}\right)=s\left(\bar{h}_{2}\right)$, then

(a) If $d\left(\bar{h}_{1}\right)>d\left(\bar{h}_{2}\right)$, then $\bar{h}_{1}$ is smaller than $\bar{h}_{2}$, denoted by $\bar{h}_{1}<\bar{h}_{2}$;

(b) If $d\left(\bar{h}_{1}\right)=d\left(\bar{h}_{2}\right)$, then $\bar{h}_{1}$ and $\bar{h}_{2}$ represent the same information, denoted by $\bar{h}_{1}=\bar{h}_{2}$.

Some operations to aggregate HPFEs based on the operations of HFEs [12,13] are defined as follows:

Definition 4. [27] Let $\bar{h}\left(\gamma_{i} \mid p_{i}\right), \bar{h}_{1}\left(\dot{\gamma}_{j} \mid \dot{p}_{j}\right)$ and $\bar{h}_{2}\left(\ddot{\gamma}_{k} \mid \ddot{p}_{k}\right)$ be three HPFEs, $i=1,2, \ldots, \# \bar{h}, j=1,2, \ldots, \# \bar{h}_{1}$, $k=1,2, \ldots, \# \bar{h}_{2}$, and $\lambda>0$, then

(1) $(\bar{h})^{c}=\cup_{i=1,2, \ldots, \# \bar{h}}\left\{\left(1-\gamma_{i}\right) \mid p_{i}\right\}$;

(2) $\lambda \bar{h}=\cup_{i=1,2, \ldots, \# \bar{h}}\left\{1-\left(1-\gamma_{i}\right)^{\lambda} \mid p_{i}\right\}$;

(3) $\bar{h}^{\lambda}=\cup_{i=1,2, \ldots, \# \bar{h}}\left\{\left(\gamma_{i}\right)^{\lambda} \mid p_{i}\right\}$;

(4) $\bar{h}_{1} \oplus \bar{h}_{2}=\cup_{j=1,2, \ldots, \# \bar{h}_{1}, k=1,2, \ldots, \# \bar{h}_{2}}\left\{\left(\dot{\gamma}_{j}+\ddot{\gamma}_{k}-\dot{\gamma}_{j} \ddot{\gamma}_{k}\right) \mid \dot{p}_{j} \ddot{p}_{k}\right\}$;

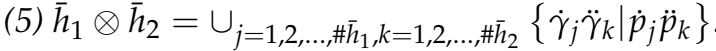

Theorem 1. Let $\bar{h}\left(\gamma_{i} \mid p_{i}\right), \bar{h}_{1}\left(\dot{\gamma}_{j} \mid \dot{p}_{j}\right)$ and $\bar{h}_{2}\left(\ddot{\gamma}_{k} \mid \ddot{p}_{k}\right)$ be three HPFEs, $i=1,2, \ldots, \# \bar{h}, j=1,2, \ldots, \# \bar{h}_{1}$, $k=1,2, \ldots, \# \bar{h}_{2}, \lambda>0, \lambda_{1}>0$, and $\lambda_{2}>0$, then

(1) $\bar{h}_{1} \oplus \bar{h}_{2}=\bar{h}_{2} \oplus \bar{h}_{1}$;

(2) $\bar{h} \oplus\left(\bar{h}_{1} \oplus \bar{h}_{2}\right)=\left(\bar{h} \oplus \bar{h}_{1}\right) \oplus \bar{h}_{2}$;

(3) $\lambda\left(\bar{h}_{1} \oplus \bar{h}_{2}\right)=\left(\lambda \bar{h}_{1}\right) \oplus\left(\lambda \bar{h}_{2}\right)$;

(4) $\lambda_{1}\left(\lambda_{2} \bar{h}\right)=\left(\lambda_{1} \lambda_{2}\right) \bar{h}$; 
(5) $\bar{h}_{1} \otimes \bar{h}_{2}=\bar{h}_{2} \otimes \bar{h}_{1}$

(6) $\bar{h} \otimes\left(\bar{h}_{1} \otimes \bar{h}_{2}\right)=\left(\bar{h} \otimes \bar{h}_{1}\right) \otimes \bar{h}_{2} ;$

(7) $\left(\bar{h}_{1} \otimes \bar{h}_{2}\right)^{\lambda}=\bar{h}_{1}^{\lambda} \otimes \bar{h}_{2}^{\lambda}$;

(8) $\left(\bar{h}^{\lambda_{1}}\right)^{\lambda_{2}}=\bar{h}^{\left(\lambda_{1} \lambda_{2}\right)}$.

Proof. We only prove (3) and the other are trivial or similar to (3).

(3) Since $\bar{h}_{1} \oplus \bar{h}_{2}=\cup_{j=1,2, \ldots, \# \bar{h}_{1}, k=1,2, \ldots, \# \bar{h}_{2}}\left\{\dot{\gamma}_{j}+\ddot{\gamma}_{k}-\dot{\gamma}_{j} \ddot{\gamma}_{k} \mid \dot{p}_{j} \ddot{p}_{k}\right\}$, according to the operational law (2) in Definition 4, we have

$$
\begin{aligned}
\lambda\left(\bar{h}_{1} \oplus \bar{h}_{2}\right) & =\cup_{\substack{j=1,2, \ldots, \ldots \bar{h}_{1}, k=1,2, \ldots, \ldots \bar{h}_{2}}}\left\{1-\left(1-\left(\dot{\gamma}_{j}+\ddot{\gamma}_{k}-\dot{\gamma}_{j} \ddot{\gamma}_{k}\right)\right)^{\lambda} \mid \dot{p}_{j} \ddot{p}_{k}\right\} \\
& =\cup_{\substack{j=1,2, \ldots, \ldots \bar{h}_{1}, k=1,2, \ldots, \ldots h_{2}}}\left\{1-\left(\left(1-\dot{\gamma}_{j}\right)\left(1-\ddot{\gamma}_{k}\right)\right)^{\lambda} \mid \dot{p}_{j} \ddot{p}_{k}\right\} .
\end{aligned}
$$

Since $\lambda \bar{h}_{1}=\cup_{j=1,2, \ldots, \# \bar{h}_{1}}\left\{1-\left(1-\dot{\gamma}_{j}\right)^{\lambda} \mid \dot{p}_{j}\right\}$ and $\lambda \bar{h}_{2}=\cup_{k=1,2, \ldots, \# \bar{h}_{2}}\left\{1-\left(1-\dot{\gamma}_{k}\right)^{\lambda} \mid \ddot{p}_{k}\right\}$, we have

$$
\begin{aligned}
\left(\lambda \bar{h}_{1}\right) \oplus\left(\lambda \bar{h}_{2}\right) & =\cup_{\substack{j=1,2, \ldots, \ldots \bar{h}_{1}, k=1,2, \ldots, \bar{h}_{2}}}\left\{1-\left(1-\dot{\gamma}_{j}\right)^{\lambda}+1-\left(1-\dot{\gamma}_{k}\right)^{\lambda}-\left(1-\left(1-\dot{\gamma}_{j}\right)^{\lambda}\right)\left(1-\left(1-\dot{\gamma}_{k}\right)^{\lambda}\right) \mid \dot{p}_{j} \ddot{p}_{k}\right\} \\
& =\cup_{\substack{j=1,2, \ldots, \ldots \bar{h}_{1}, k=1,2, \ldots, \ldots h_{2}}}\left\{1-\left(1-\dot{\gamma}_{j}\right)^{\lambda}\left(1-\ddot{\gamma}_{k}\right)^{\lambda} \mid \dot{p}_{j} \ddot{p}_{k}\right\} .
\end{aligned}
$$

Hence, $\lambda\left(\bar{h}_{1} \oplus \bar{h}_{2}\right)=\left(\lambda \bar{h}_{1}\right) \oplus\left(\lambda \bar{h}_{2}\right)$.

However, for an HPFE $\bar{h}\left(\gamma_{i} \mid p_{i}\right), i=1,2, \ldots, \# \bar{h}, \lambda_{1}>0$ and $\lambda_{2}>0$, the operational laws $\left(\lambda_{1} \bar{h}\right) \oplus\left(\lambda_{2} \bar{h}\right)=\left(\lambda_{1}+\lambda_{2}\right) \bar{h}$ and $\bar{h}^{\lambda_{1}} \otimes \bar{h}^{\lambda_{2}}=\bar{h}^{\left(\lambda_{1}+\lambda_{2}\right)}$ do not hold in general. To illustrate this case, we give the following example.

Example 1. Let $\bar{h}\left(\gamma_{i} \mid p_{i}\right)=(0.7|0.5,0.2| 0.5)$ and $\lambda_{1}=\lambda_{2}=1$, then

$$
\begin{aligned}
&\left(\lambda_{1} \bar{h}\right) \oplus\left(\lambda_{2} \bar{h}\right)=\bar{h} \oplus \bar{h}=\cup_{i, j=1,2}\left\{\gamma_{i}+\gamma_{j}-\gamma_{i} \gamma_{j} \mid 0.25\right\} \\
&=(0.91|0.25,0.76| 0.25,0.76|0.25,0.36| 0.25) \\
&\left(\lambda_{1}+\lambda_{2}\right) \bar{h}=2 \bar{h}=\cup_{i=1,2}\left\{1-\left(1-\gamma_{i}\right)^{2} \mid 0.5\right\}=(0.91|0.5,0.36| 0.5)
\end{aligned}
$$

and $s\left(\left(\lambda_{1} \bar{h}\right) \oplus\left(\lambda_{2} \bar{h}\right)\right)=0.6975>0.635=s\left(\left(\lambda_{1}+\lambda_{2}\right) \bar{h}\right)$ and hence, $\left(\lambda_{1} \bar{h}\right) \oplus\left(\lambda_{2} \bar{h}\right)>\left(\lambda_{1}+\lambda_{2}\right) \bar{h}$. Similarly, we have $s\left(\bar{h}^{\lambda_{1}} \otimes \bar{h}^{\lambda_{2}}\right)=0.2025<0.265=s\left(\bar{h}^{\left(\lambda_{1}+\lambda_{2}\right)}\right)$ and thus, $\bar{h}^{\lambda_{1}} \otimes \bar{h}^{\lambda_{2}}<\bar{h}^{\left(\lambda_{1}+\lambda_{2}\right)}$.

Based on Definition 4, in order to aggregate the HPFEs, $X u$ and Zhou [27] developed some hesitant probabilistic fuzzy aggregation operators, as follows:

Definition 5. [27] Let $\bar{h}_{t}(t=1,2, \ldots, T)$ be a collection of HPFEs, $w=\left(w_{1}, w_{2}, \ldots, w_{T}\right)^{T}$ be the weight vector of $\bar{h}_{t}$ with $w_{t} \in[0,1]$, and $\sum_{t=1}^{T} w_{t}=1$, and $p_{t}$ be the probability of $\gamma_{t}$ in the HPFE $\bar{h}_{t}$, then

(1) the hesitant probabilistic fuzzy weighted averaging (HPFWA) operator is

$$
\begin{aligned}
\operatorname{HPFWA}\left(\bar{h}_{1}, \bar{h}_{2}, \ldots, \bar{h}_{T}\right) & =\left(w_{1} \bar{h}_{1}\right) \oplus\left(w_{2} \bar{h}_{2}\right) \oplus \cdots \oplus\left(w_{T} \bar{h}_{T}\right) \\
& =\cup_{\gamma_{1} \in \bar{h}_{1}, \gamma_{2} \in \bar{h}_{2}, \ldots, \gamma_{T} \in \bar{h}_{T}}\left\{1-\prod_{t=1}^{T}\left(1-\gamma_{t}\right)^{w_{t}} \mid p_{1} p_{2} \cdots p_{T}\right\} .
\end{aligned}
$$


(2) the hesitant probabilistic fuzzy weighted geometric (HPFWG) operator is

$$
\begin{aligned}
\operatorname{HPFWG}\left(\bar{h}_{1}, \bar{h}_{2}, \ldots, \bar{h}_{T}\right) & =\left(\bar{h}_{1}\right)^{w_{1}} \otimes\left(\bar{h}_{2}\right)^{w_{2}} \otimes \cdots \otimes\left(\bar{h}_{T}\right)^{w_{T}} \\
& =\cup_{\gamma_{1} \in \bar{h}_{1}, \gamma_{2} \in \bar{h}_{2}, \ldots, \gamma_{T} \in \bar{h}_{T}}\left\{\prod_{t=1}^{T}\left(\gamma_{t}\right)^{w_{t}} \mid p_{1} p_{2} \cdots p_{T}\right\} .
\end{aligned}
$$

Definition 6. [27] Let $\bar{h}_{t}(t=1,2, \ldots, T)$ be a collection of HPFEs, $\bar{h}_{\sigma(t)}$ be the th largest of $\bar{h}_{t}$ $(t=1,2, \ldots, T)$, and $p_{\sigma(t)}$ be the probability of $\gamma_{\sigma(t)}$ in the HPFE $\bar{h}_{\sigma(t)}$, then the following two aggregation operators, which are based on the mapping $H_{P}^{T} \rightarrow H_{P}$ with an associated vector $\omega=\left(\omega_{1}, \omega_{2}, \ldots, \omega_{T}\right)^{T}$ such that $\omega_{t} \in[0,1]$ and $\sum_{t=1}^{T} \omega_{t}=1$, are given by

(1) the hesitant probabilistic fuzzy ordered weighted averaging (HPFOWA) operator:

$$
\begin{aligned}
\operatorname{HPFOWA}\left(\bar{h}_{1}, \bar{h}_{2}, \ldots, \bar{h}_{T}\right)=\left(\omega_{1} \bar{h}_{\sigma(1)}\right) \oplus\left(\omega_{2} \bar{h}_{\sigma(2)}\right) \oplus \cdots \oplus\left(\omega_{T} \bar{h}_{\sigma(T)}\right) \\
=\cup_{\gamma_{\sigma(1)} \in \bar{h}_{\sigma(1)}, \gamma_{\sigma(2)} \in \bar{h}_{\sigma(2)}, \ldots, \gamma_{\sigma(T)} \in \bar{h}_{\sigma(T)}}\left\{1-\prod_{t=1}^{T}\left(1-\gamma_{\sigma(t)}\right)^{w_{t}} \mid p_{\sigma(1)} p_{\sigma(2)} \cdots p_{\sigma(T)}\right\} .
\end{aligned}
$$

(2) the hesitant probabilistic fuzzy ordered weighted geometric (HPFOWG) operator:

$$
\begin{aligned}
& \operatorname{HPFOWG}\left(\bar{h}_{1}, \bar{h}_{2}, \ldots, \bar{h}_{T}\right)=\left(\bar{h}_{\sigma(1)}\right)^{\omega_{1}} \otimes\left(\bar{h}_{\sigma(2)}\right)^{\omega_{2}} \otimes \cdots \otimes\left(\bar{h}_{\sigma(T)}\right)^{\omega_{T}} \\
& =\cup_{\gamma_{\sigma(1)} \in \bar{h}_{\sigma(1)}, \gamma_{\sigma(2)} \in \bar{h}_{\sigma(2)}, \cdots, \gamma_{\sigma(T)} \in \bar{h}_{\sigma(T)}}\left\{\prod_{t=1}^{T}\left(\gamma_{\sigma(t)}\right)^{w_{t}} \mid p_{\sigma(1)} p_{\sigma(2)} \cdots p_{\sigma(T)}\right\} .
\end{aligned}
$$

\subsection{Einstein Operations on HPFEs}

It is well known that the $t$-norms and $t$-conorms are general concepts satisfying the requirements of the conjunction and disjunction operators. Einstein operations include the Einstein sum $\left(\oplus_{\varepsilon}\right)$ and Einstein product $\left(\otimes_{\mathcal{E}}\right)$ which are examples of $t$-conorms and $t$-norms, respectively. They were defined by Klement et al. [35] as follows:

$$
x \otimes_{\varepsilon} y=\frac{x y}{1+(1-x)(1-y)}, x \oplus_{\varepsilon} y=\frac{x+y}{1+x y}, x, y \in[0,1] .
$$

Based on the above Einstein operations, we give the following new operations on HPFEs:

Definition 7. Let $\bar{h}\left(\gamma_{i} \mid p_{i}\right), \bar{h}_{1}\left(\dot{\gamma}_{j} \mid \dot{p}_{j}\right)$ and $\bar{h}_{2}\left(\ddot{\gamma}_{k} \mid \ddot{p}_{k}\right)$ be three HPFEs, $i=1,2, \ldots, \# \bar{h}, j=1,2, \ldots, \# \bar{h}_{1}$, $k=1,2, \ldots, \# \bar{h}_{2}$, and $\lambda>0$, then

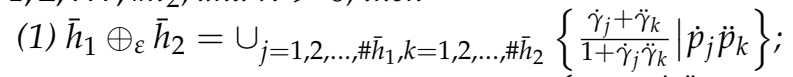

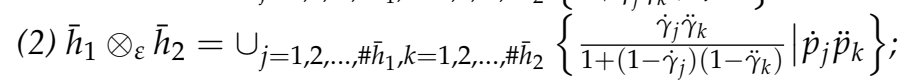

$$
\begin{aligned}
& \text { (3) } \lambda \cdot \varepsilon \bar{h}=\cup_{i=1,2, \ldots, \# \bar{h}}\left\{\frac{\left(1+\gamma_{i}\right)^{\lambda}-\left(1-\gamma_{i}\right)^{\lambda}}{\left(1+\gamma_{i}\right)^{\lambda}+\left(1-\gamma_{i}\right)^{\lambda}} \mid p_{i}\right\} \text {; } \\
& \text { (4) } \bar{h}^{\wedge} \lambda=\cup_{i=1,2, \ldots, \# \bar{h}}\left\{\frac{2 \gamma_{i}^{\lambda}}{\left(2-\gamma_{i}\right)^{\lambda}+\gamma_{i}^{\lambda}} \mid p_{i}\right\} \text {. }
\end{aligned}
$$

Thus, the above four operations on the HPFEs can be suitable for the HPFSs. Moreover, some relationships are discussed for the operations on HPFEs given in Definitions 4 and 7 as follows:

Theorem 2. Let $\bar{h}\left(\gamma_{i} \mid p_{i}\right), \bar{h}_{1}\left(\dot{\gamma}_{j} \mid \dot{p}_{j}\right)$ and $\bar{h}_{2}\left(\ddot{\gamma}_{k} \mid \ddot{p}_{k}\right)$ be three HPFEs, $i=1,2, \ldots, \# \bar{h}, j=1,2, \ldots, \# \bar{h}_{1}$, $k=1,2, \ldots, \# \bar{h}_{2}$, and $\lambda>0$, then
(1) $\left((\bar{h})^{c}\right)^{\wedge_{\varepsilon} \lambda}=(\lambda \cdot \varepsilon \bar{h})^{c}$;
(2) $\lambda \cdot \varepsilon(\bar{h})^{c}=\left(\bar{h}^{\wedge_{\varepsilon} \lambda}\right)^{c}$;
(3) $\left(\bar{h}_{1}\right)^{c} \oplus_{\varepsilon}\left(\bar{h}_{2}\right)^{c}=\left(\bar{h}_{1} \otimes_{\varepsilon} \bar{h}_{2}\right)^{c}$;
(4) $\left(\bar{h}_{1}\right)^{c} \otimes_{\varepsilon}\left(\bar{h}_{2}\right)^{c}=\left(\bar{h}_{1} \oplus_{\varepsilon} \bar{h}_{2}\right)^{c}$. 
Proof. (1)

$$
\begin{aligned}
\left((\bar{h})^{c}\right)^{\wedge_{\varepsilon} \lambda} & =\cup_{i=1,2, \ldots, \# \bar{h}}\left\{\frac{2\left(1-\gamma_{i}\right)^{\lambda}}{\left(2-\left(1-\gamma_{i}\right)\right)^{\lambda}+\left(1-\gamma_{i}\right)^{\lambda}} \mid p_{i}\right\} \\
& =\cup_{i=1,2, \ldots, \# \bar{h}}\left\{\frac{2\left(1-\gamma_{i}\right)^{\lambda}}{\left.\left(1+\gamma_{i}\right)\right)^{\lambda}+\left(1-\gamma_{i}\right)^{\lambda}} \mid p_{i}\right\} \\
& =\left(\cup_{i=1,2, \ldots, \# \bar{h}}\left\{\frac{\left(1+\gamma_{i}\right)^{\lambda}-\left(1-\gamma_{i}\right)^{\lambda}}{\left.\left(1+\gamma_{i}\right)\right)^{\lambda}+\left(1-\gamma_{i}\right)^{\lambda}} \mid p_{i}\right\}\right)^{c} \\
& =\left(\lambda \cdot{ }_{\varepsilon} \bar{h}\right)^{c} .
\end{aligned}
$$

(2)

$$
\begin{aligned}
\lambda \cdot_{\varepsilon}(\bar{h})^{c} & =\cup_{i=1,2, \ldots, \ldots \hbar h}\left\{\frac{\left(1+\left(1-\gamma_{i}\right)\right)^{\lambda}-\left(1-\left(1-\gamma_{i}\right)\right)^{\lambda}}{\left(1+\left(1-\gamma_{i}\right)\right)^{\lambda}+\left(1-\left(1-\gamma_{i}\right)\right)^{\lambda}} \mid p_{i}\right\} \\
& =\cup_{i=1,2, \ldots, \ldots \bar{h}}\left\{1-\frac{2 \gamma_{i}^{\lambda}}{\left(2-\gamma_{i}\right)^{\lambda}+\gamma_{i}^{\lambda}} \mid p_{i}\right\} \\
& =\left(\cup_{i=1,2, \ldots, \# \bar{h}}\left\{\frac{2 \gamma_{i}^{\lambda}}{\left(2-\gamma_{i}\right)^{\lambda}+\gamma_{i}^{\lambda}} \mid p_{i}\right\}\right)^{c} \\
& =\left(\bar{h}^{\wedge} \lambda\right)^{c} .
\end{aligned}
$$

(3)

$$
\begin{aligned}
& \left(\bar{h}_{1}\right)^{c} \oplus_{\mathcal{E}}\left(\bar{h}_{2}\right)^{c}=\cup_{i=1,2, \ldots, \# \bar{h}_{1}}\left\{\left(1-\dot{\gamma}_{j}\right) \mid \dot{p}_{j}\right\} \oplus_{\varepsilon} \cup_{i=1,2, \ldots, \# \bar{h}_{2}}\left\{\left(1-\ddot{\gamma}_{k}\right) \mid \ddot{p}_{k}\right\} \\
& =\cup_{\substack{j=1,2, \ldots, \# \overline{1}_{1}, k=1,2, \ldots, \mathbb{h}_{2}}}\left\{\left.\frac{\left(1-\dot{\gamma}_{j}\right)+\left(1-\ddot{\gamma}_{k}\right)}{1+\left(1-\dot{\gamma}_{j}\right)\left(1-\ddot{\gamma}_{k}\right)}\right|_{\dot{p}_{j}} \ddot{p}_{k}\right\} \\
& =\left(\cup_{\substack{j=1,2, \ldots, \ldots \overline{1}_{1,} \\
k=1,2, \ldots, \|_{h_{2}}}}\left\{\frac{\dot{\gamma}_{j} \ddot{\gamma}_{k}}{1+\left(1-\dot{\gamma}_{j}\right)\left(1-\ddot{\gamma}_{k}\right)} \mid \dot{p}_{j} \ddot{p}_{k}\right\}\right)^{c} \\
& =\left(\bar{h}_{1} \otimes_{\varepsilon} \bar{h}_{2}\right)^{c} \text {. }
\end{aligned}
$$

(4)

$$
\begin{aligned}
& \left(\bar{h}_{1}\right)^{\mathcal{c}} \otimes_{\mathcal{E}}\left(\bar{h}_{2}\right)^{\mathcal{c}}=\cup_{i=1,2, \ldots, \ldots \bar{h}_{1}}\left\{\left(1-\dot{\gamma}_{j}\right) \mid \dot{p}_{j}\right\} \otimes_{\mathcal{E}} \cup_{i=1,2, \ldots, \# \bar{h}_{2}}\left\{\left(1-\ddot{\gamma}_{k}\right) \mid \ddot{p}_{k}\right\} \\
& =\cup_{\substack{j=1,2, \ldots, \ldots \overline{1}_{1}, k=1,2, \ldots, \ldots \bar{h}_{2}}}\left\{\frac{\left(1-\dot{\gamma}_{j}\right)\left(1-\ddot{\gamma}_{k}\right)}{1-\dot{\gamma}_{j} \ddot{\gamma}_{k}} \mid \dot{p}_{j} \ddot{p}_{k}\right\} \\
& =\left(\cup_{\substack{j=1,2, \ldots, \ldots \overline{1}_{1,} \\
k=1,2, \ldots, \ldots \bar{h}_{2}}}\left\{\frac{\dot{\gamma}_{j}+\ddot{\gamma}_{k}}{1+\dot{\gamma}_{j} \ddot{\gamma}_{k}} \mid \dot{p}_{j} \ddot{p}_{k}\right\}\right)^{c} \\
& =\left(\bar{h}_{1} \oplus_{\varepsilon} \bar{h}_{2}\right)^{c} \text {. }
\end{aligned}
$$

Theorem 3. Let $\bar{h}\left(\gamma_{i} \mid p_{i}\right), \bar{h}_{1}\left(\dot{\gamma}_{j} \mid \dot{p}_{j}\right)$ and $\bar{h}_{2}\left(\ddot{\gamma}_{k} \mid \ddot{p}_{k}\right)$ be three HPFEs, $i=1,2, \ldots, \# \bar{h}, j=1,2, \ldots, \# \bar{h}_{1}$, $k=1,2, \ldots, \# \bar{h}_{2}, \lambda>0, \lambda_{1}>0$, and $\lambda_{2}>0$, then

(1) $\bar{h}_{1} \oplus_{\varepsilon} \bar{h}_{2}=\bar{h}_{2} \oplus_{\varepsilon} \bar{h}_{1}$;

(2) $\bar{h} \oplus_{\varepsilon}\left(\bar{h}_{1} \oplus_{\varepsilon} \bar{h}_{2}\right)=\left(\bar{h} \oplus_{\varepsilon} \bar{h}_{1}\right) \oplus_{\varepsilon} \bar{h}_{2}$;

(3) $\lambda \cdot \cdot_{\varepsilon}\left(\bar{h}_{1} \oplus_{\varepsilon} \bar{h}_{2}\right)=\left(\lambda \cdot{ }_{\varepsilon} \bar{h}_{1}\right) \oplus_{\varepsilon}\left(\lambda \cdot{ }_{\varepsilon} \bar{h}_{2}\right)$;

(4) $\lambda_{1} \cdot \varepsilon\left(\lambda_{2} \cdot \varepsilon \bar{h}\right)=\left(\lambda_{1} \lambda_{2}\right) \cdot \varepsilon \bar{h}$;

(5) $\bar{h}_{1} \otimes_{\mathcal{E}} \bar{h}_{2}=\bar{h}_{2} \otimes_{\varepsilon} \bar{h}_{1}$; 
(6) $\bar{h} \otimes_{\varepsilon}\left(\bar{h}_{1} \otimes_{\varepsilon} \bar{h}_{2}\right)=\left(\bar{h} \otimes_{\varepsilon} \bar{h}_{1}\right) \otimes_{\varepsilon} \bar{h}_{2}$

(7) $\left(\bar{h}_{1} \otimes_{\varepsilon} \bar{h}_{2}\right)^{\wedge_{\varepsilon} \lambda}=\bar{h}_{1}^{\wedge_{\varepsilon} \lambda} \otimes_{\varepsilon} \bar{h}_{2}^{\wedge_{\varepsilon} \lambda}$;

(8) $\left(\bar{h}^{\wedge_{\varepsilon} \lambda_{1}}\right)^{\wedge_{\varepsilon} \lambda_{2}}=\bar{h}^{\wedge_{\varepsilon}\left(\lambda_{1} \lambda_{2}\right)}$.

Proof. Since (1), (2), (5) and (6) are trivial, and (7) and (8) are similar to (3) and (4), respectively, we only prove (3) and (4).

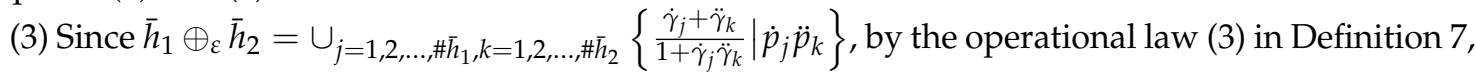
we have

$$
\begin{aligned}
& \lambda \cdot \varepsilon\left(\bar{h}_{1} \oplus_{\varepsilon} \bar{h}_{2}\right)=\bigcup_{\substack{j=1,2, \ldots, \ldots h_{1}, k=1,2, \ldots, \bar{h}_{2}}}\left\{\frac{\left(1+\frac{\dot{\gamma}_{j}+\ddot{\gamma}_{k}}{1+\dot{\gamma}_{j} \ddot{\gamma}_{k}}\right)^{\lambda}-\left(1-\frac{\dot{\gamma}_{j}+\ddot{\gamma}_{k}}{1+\dot{\gamma}_{j} \ddot{\gamma}_{k}}\right)^{\lambda}}{\left(1+\frac{\dot{\gamma}_{j}+\dot{\gamma}_{k}}{1+\dot{\gamma}_{j} \ddot{\gamma}_{k}}\right)^{\lambda}+\left(1-\frac{\dot{\gamma}_{j}+\ddot{\gamma}_{k}}{1+\dot{\gamma}_{j} \ddot{\gamma}_{k}}\right)^{\lambda}} \dot{p}_{j} \ddot{p}_{k}\right\} \\
& =\bigcup_{\substack{j=1,2, \ldots, \ldots \bar{h}_{1}, k=1,2, \ldots, \ldots \bar{h}_{2}}}\left\{\frac{\left(1+\dot{\gamma}_{j}\right)^{\lambda}\left(1+\ddot{\gamma}_{k}\right)^{\lambda}-\left(1-\dot{\gamma}_{j}\right)^{\lambda}\left(1-\ddot{\gamma}_{k}\right)^{\lambda}}{\left(1+\dot{\gamma}_{j}\right)^{\lambda}\left(1+\ddot{\gamma}_{k}\right)^{\lambda}+\left(1-\dot{\gamma}_{j}\right)^{\lambda}\left(1-\ddot{\gamma}_{k}\right)^{\lambda}} \mid \dot{p}_{j} \ddot{p}_{k}\right\} .
\end{aligned}
$$

Since $\lambda \cdot{ }_{\varepsilon} \bar{h}_{1}=\cup_{i=1,2, \ldots, \# \bar{h}_{1}}\left\{\frac{\left(1+\dot{\gamma}_{j}\right)^{\lambda}-\left(1-\dot{\gamma}_{j}\right)^{\lambda}}{\left(1+\dot{\gamma}_{j}\right)^{\lambda}+\left(1-\dot{\gamma}_{j}\right)^{\lambda}} \dot{p}_{j}\right\}$ and $\lambda \cdot{ }_{\varepsilon} \bar{h}_{2}=\cup_{i=1,2, \ldots, \# \bar{h}_{2}}\left\{\frac{\left(1+\ddot{\gamma}_{k}\right)^{\lambda}-\left(1-\ddot{\gamma}_{k}\right)^{\lambda}}{\left(1+\ddot{\gamma}_{k}\right)^{\lambda}+\left(1-\ddot{\gamma}_{k}\right)^{\lambda}} \mid \ddot{p}_{k}\right\}$, we have

$$
\begin{aligned}
\left(\lambda \cdot \bar{h}_{1}\right) \oplus_{\varepsilon}\left(\lambda \cdot \bar{h}_{2}\right) & =\bigcup_{\substack{j=1,2, \ldots, \ldots \bar{h}_{1}, k=1,2, \ldots, \bar{h}_{2}}}\left\{\frac{\frac{\left(1+\dot{\gamma}_{j}\right)^{\lambda}-\left(1-\dot{\gamma}_{j}\right)^{\lambda}}{\left(1+\dot{\gamma}_{j}\right)^{\lambda}+\left(1-\dot{\gamma}_{j}\right)^{\lambda}}+\frac{\left(1+\ddot{\gamma}_{k}\right)^{\lambda}-\left(1-\ddot{\gamma}_{k}\right)^{\lambda}}{\left(1+\dot{\gamma}_{k}\right)^{\lambda}+\left(1-\dot{\gamma}_{k}\right)^{\lambda}}}{1+\frac{\left(1+\dot{\gamma}_{j}\right)^{\lambda}-\left(1-\dot{\gamma}_{j}\right)^{\lambda}}{\left(1+\dot{\gamma}_{j}\right)^{\lambda}+\left(1-\dot{\gamma}_{j}\right)^{\lambda}} \cdot \frac{\left(1+\ddot{\gamma}_{k}\right)^{\lambda}-\left(1-\ddot{\gamma}_{k}\right)^{\lambda}}{\left(1+\ddot{\gamma}_{k}\right)^{\lambda}+\left(1-\dot{\gamma}_{k}\right)^{\lambda}}} \dot{p}_{j} \ddot{p}_{k}\right\} \\
& =\bigcup_{\substack{j=1,2, \ldots, \ldots \bar{h}_{1}, k=1,2, \ldots, \ldots \bar{h}_{2}}}\left\{\frac{\left(1+\dot{\gamma}_{j}\right)^{\lambda}\left(1+\ddot{\gamma}_{k}\right)^{\lambda}-\left(1-\dot{\gamma}_{j}\right)^{\lambda}\left(1-\ddot{\gamma}_{k}\right)^{\lambda}}{\left(1+\dot{\gamma}_{j}\right)^{\lambda}\left(1+\ddot{\gamma}_{k}\right)^{\lambda}+\left(1-\dot{\gamma}_{j}\right)^{\lambda}\left(1-\ddot{\gamma}_{k}\right)^{\lambda}} \mid \dot{p}_{j} \ddot{p}_{k}\right\} .
\end{aligned}
$$

Hence $\lambda \cdot \cdot_{\varepsilon}\left(\bar{h}_{1} \oplus_{\varepsilon} \bar{h}_{2}\right)=\left(\lambda \cdot{ }_{\varepsilon} \bar{h}_{1}\right) \oplus_{\varepsilon}\left(\lambda \cdot{ }_{\varepsilon} \bar{h}_{2}\right)$.

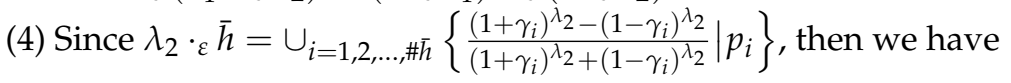

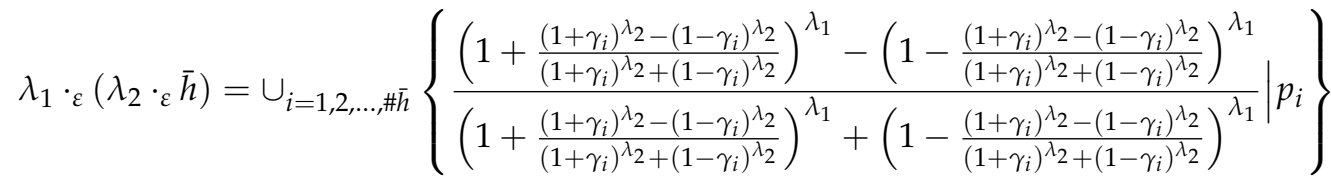

$$
\begin{aligned}
& =\cup_{i=1,2, \ldots, \# \bar{h}}\left\{\frac{\left(1+\gamma_{i}\right)^{\left(\lambda_{1} \lambda_{2}\right)}-\left(1-\gamma_{i}\right)^{\left(\lambda_{1} \lambda_{2}\right)}}{\left(1+\gamma_{i}\right)^{\left(\lambda_{1} \lambda_{2}\right)}+\left(1-\gamma_{i}\right)^{\left(\lambda_{1} \lambda_{2}\right)}} \mid p_{i}\right\} \\
& =\left(\lambda_{1} \lambda_{2}\right) \cdot{ }_{\varepsilon} \bar{h} .
\end{aligned}
$$

For an HPFE, $\bar{h}\left(\gamma_{i} \mid p_{i}\right), i=1,2, \ldots, \# \bar{h}, \lambda_{1}>0$, and $\lambda_{2}>0$, the operational laws $\left(\lambda_{1} \cdot \varepsilon \bar{h}\right) \oplus_{\varepsilon}\left(\lambda_{2} \cdot \varepsilon\right.$ $\bar{h})=\left(\lambda_{1}+\lambda_{2}\right) \cdot{ }_{\varepsilon} \bar{h}$ and $\bar{h}^{\wedge_{\varepsilon} \lambda_{1}} \otimes_{\mathcal{E}} \bar{h}^{\wedge_{\varepsilon} \lambda_{2}}=\bar{h}^{\wedge_{\varepsilon}\left(\lambda_{1}+\lambda_{2}\right)}$ do not hold in general. To illustrate this case, we give the following example.

Example 2. Let $\bar{h}\left(\gamma_{i} \mid p_{i}\right)=(0.3|0.5,0.5| 0.5)$ and $\lambda_{1}=\lambda_{2}=1$, then

$$
\begin{aligned}
\left(\lambda_{1} \cdot{ }_{\varepsilon} \bar{h}\right) \oplus_{\varepsilon}\left(\lambda_{2} \cdot \bar{\varepsilon} \bar{h}\right) & =\bar{h} \oplus_{\varepsilon} \bar{h}=\cup_{i, j=1,2}\left\{\frac{\gamma_{i}+\gamma_{j}}{1+\gamma_{i} \gamma_{j}} \mid 0.25\right\} \\
& =(0.5505|0.25,0.6957| 0.25,0.6957|0.25,0.8| 0.25)
\end{aligned}
$$




$$
\begin{aligned}
\left(\lambda_{1}+\lambda_{2}\right) \cdot{ }_{\varepsilon} \bar{h} & =2 \cdot{ }_{\varepsilon} \bar{h}=\cup_{i=1,2}\left\{\frac{\left(1+\gamma_{i}\right)^{2}-\left(1-\gamma_{i}\right)^{2}}{\left(1+\gamma_{i}\right)^{2}+\left(1-\gamma_{i}\right)^{2}} \mid 0.5\right\} \\
& =(0.5505|0.5,0.8| 0.5) .
\end{aligned}
$$

Clearly, $s\left(\left(\lambda_{1} \cdot{ }_{\varepsilon} \bar{h}\right) \oplus_{\varepsilon}\left(\lambda_{2} \cdot \varepsilon \bar{h}\right)\right)=0.6856>0.6752=s\left(\left(\lambda_{1}+\lambda_{2}\right) \cdot_{\varepsilon} \bar{h}\right)$. Hence, $\left(\lambda_{1} \cdot \varepsilon \bar{h}\right) \oplus_{\varepsilon}\left(\lambda_{2} \cdot \varepsilon\right.$ $\bar{h})<\left(\lambda_{1}+\lambda_{2}\right) \cdot \cdot_{\varepsilon} \bar{h}$. Similarly, we have $s\left(\bar{h}^{\wedge_{\varepsilon} \lambda_{1}} \otimes_{\varepsilon} \bar{h}^{\wedge_{\varepsilon} \lambda_{2}}\right)=0.2566>0.13=s\left(\bar{h}^{\wedge_{\varepsilon}\left(\lambda_{1}+\lambda_{2}\right)}\right)$ and thus $\bar{h}^{\wedge_{\varepsilon} \lambda_{1}} \otimes_{\varepsilon} \bar{h}^{\wedge_{\varepsilon} \lambda_{2}}<\bar{h}^{\wedge_{\varepsilon}\left(\lambda_{1}+\lambda_{2}\right)}$.

\section{Some HPFE Weighted Aggregation Operators Based on Einstein Operation}

One important issue is the question of how to extend Einstein operations to aggregate the HPFE information provided by the decision makers. The optimal approach is weighted aggregation operators, in which the widely used technologies are the weighted averaging (WA) operator, the ordered weighted averaging (OWA) operator, and their extended forms [39,40]. Yu [21] proposed the hesitant fuzzy Einstein weighted averaging (HFEWA) operator, the hesitant fuzzy Einstein ordered weighted averaging (HFEOWA) operator, the hesitant fuzzy Einstein weighted geometric (HFEWG) operator, and the hesitant fuzzy Einstein ordered weighted geometric (HFEOWG) operator based on those operators. Similar to these hesitant fuzzy information aggregation operators, we propose the corresponding hesitant probabilistic fuzzy Einstein weighted and ordered operators to aggregate the HPFEs.

Definition 8. Let $\bar{h}_{t}(t=1,2, \ldots, T)$ be a collection of HPFEs; then, a hesitant probabilistic fuzzy Einstein weighted averaging (HPFEWA) operator is a mapping $H_{P}^{T} \rightarrow H_{P}$ such that

$$
\operatorname{HPFEWA}\left(\bar{h}_{1}, \bar{h}_{2}, \ldots, \bar{h}_{T}\right)=\left(w_{1} \cdot \varepsilon \bar{h}_{1}\right) \oplus_{\varepsilon}\left(w_{2} \cdot \bar{h}_{2}\right) \oplus_{\varepsilon} \cdots \oplus_{\varepsilon}\left(w_{T} \cdot \bar{h}_{T}\right),
$$

where $w=\left(w_{1}, w_{2}, \ldots, w_{T}\right)^{T}$ is the weight vector of $\bar{h}_{t}(t=1,2, \ldots, T)$ with $w_{t} \in[0,1]$ and $\sum_{t=1}^{T} w_{t}=1$, and $p_{t}$ is the probability of $\gamma_{t}$ in HPFE $\bar{h}_{t}$. In particular, if $w=\left(\frac{1}{T}, \frac{1}{T}, \ldots, \frac{1}{T}\right)^{T}$, then the HPFEWA operator is reduced to the hesitant probabilistic fuzzy Einstein averaging (HPFEA) operator:

$$
\operatorname{HPFEA}\left(\bar{h}_{1}, \bar{h}_{2}, \ldots, \bar{h}_{T}\right)=\left(\frac{1}{T} \cdot{ }_{\varepsilon} \bar{h}_{1}\right) \oplus_{\mathcal{E}}\left(\frac{1}{T} \cdot{ }_{\varepsilon} \bar{h}_{2}\right) \oplus_{\mathcal{E}} \cdots \oplus_{\mathcal{E}}\left(\frac{1}{T} \cdot{ }_{\varepsilon} \bar{h}_{T}\right) .
$$

From Definitions 7 and 8, we can get the following result by using mathematical induction.

Theorem 4. Let $\bar{h}_{t}(t=1,2, \ldots, T)$ be a collection of HPFEs; then, their aggregated value obtained using the HPFEWA operator is also a HPFE, and

$$
\operatorname{HPFEWA}\left(\bar{h}_{1}, \bar{h}_{2}, \ldots, \bar{h}_{T}\right)=\bigcup_{\gamma_{1} \in \bar{h}_{1}, \gamma_{2} \in \bar{h}_{2}, \ldots, \gamma_{T} \in \bar{h}_{T}}\left\{\frac{\prod_{t=1}^{T}\left(1+\gamma_{t}\right)^{w_{t}}-\prod_{t=1}^{T}\left(1-\gamma_{t}\right)^{w_{t}}}{\prod_{t=1}^{T}\left(1+\gamma_{t}\right)^{w_{t}}+\prod_{t=1}^{T}\left(1-\gamma_{t}\right)^{w_{t}}} \mid p_{1} p_{2} \cdots p_{T}\right\}
$$

where $w=\left(w_{1}, w_{2}, \ldots, w_{T}\right)^{T}$ is the weight vector of $\bar{h}_{t}(t=1,2, \ldots, T)$ with $w_{t} \in[0,1]$ and $\sum_{t=1}^{T} w_{t}=1$, and $p_{t}$ is the probability of $\gamma_{t}$ in HPFE $\bar{h}_{t}$.

Proof. We prove Equation (8) by mathematical induction. For $T=2$, since $w_{1} \cdot_{\varepsilon} \bar{h}_{1}=$ $\cup_{\gamma_{1} \in \bar{h}_{1}}\left\{\frac{\left(1+\gamma_{1}\right)^{w_{1}}-\left(1-\gamma_{1}\right)^{w_{1}}}{\left(1+\gamma_{1}\right)^{w_{1}}+\left(1-\gamma_{1}\right)^{w_{1}}} \mid p_{1}\right\}$ and $w_{2} \cdot \varepsilon \bar{h}_{2}=\cup_{\gamma_{2} \in \bar{h}_{2}}\left\{\frac{\left(1+\gamma_{2}\right)^{w_{2}}-\left(1-\gamma_{2}\right)^{w_{2}}}{\left(1+\gamma_{2}\right)^{w_{2}}+\left(1-\gamma_{2}\right)^{w_{2}}} \mid p_{2}\right\}$, then

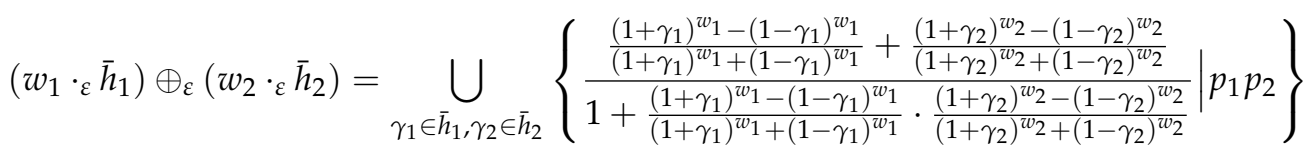

$$
\begin{aligned}
& =\bigcup_{\gamma_{1} \in \bar{h}_{1}, \gamma_{2} \in \bar{h}_{2}}\left\{\frac{\prod_{t=1}^{2}\left(1+\gamma_{t}\right)^{w_{t}}-\prod_{t=1}^{2}\left(1-\gamma_{t}\right)^{w_{t}}}{\prod_{t=1}^{2}\left(1+\gamma_{t}\right)^{w_{t}}+\prod_{t=1}^{2}\left(1-\gamma_{t}\right)^{w_{t}}} \mid p_{1} p_{2}\right\} \text {. }
\end{aligned}
$$


If Equation (8) holds for $T=k$, that is

$$
\begin{aligned}
& \left(w_{1} \cdot \bar{h}_{1}\right) \oplus_{\varepsilon}\left(w_{2} \cdot \bar{h}_{2}\right) \oplus_{\varepsilon} \cdots \oplus_{\varepsilon}\left(w_{k} \cdot \bar{h}_{k}\right) \\
& =\bigcup_{\gamma_{1} \in \bar{h}_{1}, \gamma_{2} \in \bar{h}_{2}, \ldots, \gamma_{k} \in \bar{h}_{k}}\left\{\frac{\prod_{t=1}^{k}\left(1+\gamma_{t}\right)^{w_{t}}-\prod_{t=1}^{k}\left(1-\gamma_{t}\right)^{w_{t}}}{\prod_{t=1}^{k}\left(1+\gamma_{t}\right)^{w_{t}}+\prod_{t=1}^{k}\left(1-\gamma_{t}\right)^{w_{t}}} \mid p_{1} p_{2} \cdots p_{k}\right\},
\end{aligned}
$$

then, when $T=k+1$, according to the Einstein operations of HPFEs, we have

$$
\begin{aligned}
& \left(w_{1} \cdot \bar{h}_{1}\right) \oplus_{\mathcal{E}}\left(w_{2} \cdot \bar{h}_{2}\right) \oplus_{\varepsilon} \cdots \oplus_{\varepsilon}\left(w_{k+1} \cdot \bar{h}_{k+1}\right) \\
& =\left(\left(w_{1} \cdot \bar{h}_{1}\right) \oplus_{\varepsilon}\left(w_{2} \cdot \bar{h}_{2}\right) \oplus_{\varepsilon} \cdots \oplus_{\varepsilon}\left(w_{k} \cdot \bar{h}_{k}\right)\right) \oplus_{\varepsilon}\left(w_{k+1} \cdot \bar{h}_{k+1}\right) \\
& =\bigcup_{\gamma_{1} \in \bar{h}_{1}, \gamma_{2} \in \bar{h}_{2}, \ldots, \gamma_{k} \in \bar{h}_{k}}\left\{\frac{\prod_{t=1}^{k}\left(1+\gamma_{t}\right)^{w_{t}}-\prod_{t=1}^{k}\left(1-\gamma_{t}\right)^{w_{t}}}{\prod_{t=1}^{k}\left(1+\gamma_{t}\right)^{w_{t}}+\prod_{t=1}^{k}\left(1-\gamma_{t}\right)^{w_{t}}} \mid p_{1} p_{2} \cdots p_{k}\right\} \\
& \quad \oplus_{\varepsilon} \bigcup_{\gamma_{k+1} \in \bar{h}_{k+1}}\left\{\frac{\left(1+\gamma_{k+1}\right)^{w_{k+1}}-\left(1-\gamma_{k+1}\right)^{w_{k+1}}}{\left(1+\gamma_{k+1}\right)^{w_{k+1}}+\left(1-\gamma_{k+1}\right)^{w_{k+1}}} \mid p_{k+1}\right\}
\end{aligned}
$$

i.e., Equation (8) holds for $T=k+1$. Hence, Equation (8) holds for all $T$. Thus,

$$
\operatorname{HPFEWA}\left(\bar{h}_{1}, \bar{h}_{2}, \ldots, \bar{h}_{T}\right)=\bigcup_{\gamma_{1} \in \bar{h}_{1}, \gamma_{2} \in \bar{h}_{2, \ldots, \gamma_{T} \in \bar{h}_{T}}}\left\{\frac{\prod_{t=1}^{T}\left(1+\gamma_{t}\right)^{w_{t}}-\prod_{t=1}^{T}\left(1-\gamma_{t}\right)^{w_{t}}}{\prod_{t=1}^{T}\left(1+\gamma_{t}\right)^{w_{t}}+\prod_{t=1}^{T}\left(1-\gamma_{t}\right)^{w_{t}}} \mid p_{1} p_{2} \cdots p_{T}\right\}
$$

which completes the proof of theorem.

Based on Theorem 4, we have basic properties of the HPFEWA operator, as follows:

Theorem 5. Let $\bar{h}_{t}\left(\gamma_{i}^{(t)} \mid p_{t}\right)(t=1,2, \ldots, T)$ be a collection of HPFEs, $w=\left(w_{1}, w_{2}, \ldots, w_{T}\right)^{T}$ be the weight vector of $\bar{h}_{t}(t=1,2, \ldots, T)$ such that $w_{t} \in[0,1]$ and $\sum_{t=1}^{T} w_{t}=1$, and $p_{t}$ be the corresponding probability of $\gamma_{i}^{(t)}$ in HPFE $\bar{h}_{t}$; then, we have the following:

(1) (Boundary):

$$
\bar{h}^{-} \leq \operatorname{HPFEWA}\left(\bar{h}_{1}, \bar{h}_{2}, \ldots, \bar{h}_{T}\right) \leq \bar{h}^{+},
$$

where $\bar{h}^{-}=\left(\min _{1 \leq t \leq T} \min _{\gamma_{t} \in \bar{h}_{t}} \gamma_{t} \mid p_{1} p_{2} \cdots p_{T}\right)$ and $\bar{h}^{+}=\left(\max _{1 \leq t \leq T} \max _{\gamma_{t} \in \bar{h}_{t}} \gamma_{t} \mid p_{1} p_{2} \cdots p_{T}\right)$.

(2) (Monotonicity): Let $\bar{h}_{t}^{*}\left(\dot{\gamma}_{i}^{(t)} \mid p_{t}\right)(t=1,2, \ldots, T)$ be a collection of HPFEs with $\#_{t}=\# \bar{h}_{t}=\# \bar{h}_{t}^{*}$ for $t=1,2, \ldots, T, w=\left(w_{1}, w_{2}, \ldots, w_{T}\right)^{T}$ be the weight vector of $\bar{h}_{t}^{*}(t=1,2, \ldots, T)$, such that $w_{t} \in[0,1]$ and $\sum_{t=1}^{T} w_{t}=1$, and $p_{t}$ is the probability of $\dot{\gamma}_{i}^{(t)}$ in HPFE $\bar{h}_{t}^{*}$. If $\gamma_{i}^{(t)} \leq \dot{\gamma}_{i}^{(t)}$ for each $i=1,2, \ldots, \#_{t}$, $t=1,2, \ldots, T$; then,

$$
\operatorname{HPFEWA}\left(\bar{h}_{1}, \bar{h}_{2}, \ldots, \bar{h}_{T}\right) \leq \operatorname{HPFEWA}\left(\bar{h}_{1}^{*}, \bar{h}_{2}^{*}, \ldots, \bar{h}_{T}^{*}\right) .
$$

Proof. (1) Let $f(x)=\frac{1-x}{1+x}, x \in[0,1]$, then $f^{\prime}(x)=\frac{-2}{(1+x)^{2}}<0$, i.e., $f(x)$ is a decreasing function. Let $\max \gamma_{t}=\max _{1 \leq t \leq T} \max _{\gamma_{t} \in \bar{h}_{t}} \gamma_{t}$ and $\min \gamma_{t}=\min _{1 \leq t \leq T} \min _{\gamma_{t} \in \bar{h}_{t}} \gamma_{t}$. For any $\gamma_{t} \in \bar{h}_{t}(t=1,2, \ldots, T)$, since $\min _{\gamma_{t} \in \bar{h}_{t}} \gamma_{t} \leq \gamma_{t} \leq \max _{\gamma_{t} \in \bar{h}_{t}} \gamma_{t}$, then $f\left(\max _{\gamma_{t} \in \bar{h}_{t}} \gamma_{t}\right) \leq f\left(\gamma_{t}\right) \leq f\left(\min _{\gamma_{t} \in \bar{h}_{t}} \gamma_{t}\right)$, and so

$$
\frac{1-\max \gamma_{t}}{1+\max \gamma_{t}} \leq \frac{1-\max _{\gamma_{t} \in \bar{h}_{t}} \gamma_{t}}{1+\max _{\gamma_{t} \in \bar{h}_{t}} \gamma_{t}} \leq \frac{1-\gamma_{t}}{1+\gamma_{t}} \leq \frac{1-\min _{\gamma_{t} \in \bar{h}_{t} \gamma_{t}}}{1+\min _{\gamma_{t} \in \bar{h}_{t}} \gamma_{t}} \leq \frac{1-\min \gamma_{t}}{1+\min \gamma_{t}}
$$


Since $w=\left(w_{1}, w_{2}, \ldots, w_{T}\right)^{T}$ is the weight vector of $\bar{h}_{t}(t=1,2, \ldots, T)$ with $w_{t} \in[0,1]$ and $\sum_{t=1}^{T} w_{t}=1$, we have

$$
\prod_{t=1}^{T}\left(\frac{1-\max \gamma_{t}}{1+\max \gamma_{t}}\right)^{w_{t}} \leq \prod_{t=1}^{T}\left(\frac{1-\gamma_{t}}{1+\gamma_{t}}\right)^{w_{t}} \leq \prod_{t=1}^{T}\left(\frac{1-\min \gamma_{t}}{1+\min \gamma_{t}}\right)^{w_{t}}
$$

Since $\prod_{t=1}^{T}\left(\frac{1-\max \gamma_{t}}{1+\max \gamma_{t}}\right)^{w_{t}}=\left(\frac{1-\max \gamma_{t}}{1+\max \gamma_{t}}\right)^{\sum_{t=1}^{T} w_{t}}=\frac{1-\max \gamma_{t}}{1+\max \gamma_{t}}$ and $\prod_{t=1}^{T}\left(\frac{1-\min \gamma_{t}}{1+\min \gamma_{t}}\right)^{w_{t}}=$ $\left(\frac{1-\min \gamma_{t}}{1+\min \gamma_{t}}\right)^{\sum_{t=1}^{T} w_{t}}=\frac{1-\min \gamma_{t}}{1+\min \gamma_{t}}$, we get

$$
\begin{aligned}
\frac{1-\max \gamma_{t}}{1+\max \gamma_{t}} \leq \prod_{t=1}^{T}\left(\frac{1-\gamma_{t}}{1+\gamma_{t}}\right)^{w_{t}} \leq \frac{1-\min \gamma_{t}}{1+\min \gamma_{t}} \\
\Leftrightarrow \frac{2}{1+\max \gamma_{t}} \leq 1+\prod_{t=1}^{T}\left(\frac{1-\gamma_{t}}{1+\gamma_{t}}\right)^{w_{t}} \leq \frac{2}{1+\min \gamma_{t}} \\
\Leftrightarrow \frac{1+\min \gamma_{t}}{2} \leq \frac{1}{1+\prod_{t=1}^{T}\left(\frac{1-\gamma_{t}}{1+\gamma_{t}}\right)^{w_{t}}} \leq \frac{1+\max \gamma_{t}}{2} \\
\Leftrightarrow \min \gamma_{t} \leq \frac{2}{1+\prod_{t=1}^{T}\left(\frac{1-\gamma_{t}}{1+\gamma_{t}}\right)^{w_{t}}}-1 \leq \max \gamma_{t},
\end{aligned}
$$

i.e.,

$$
\min \gamma_{t} \leq \frac{\prod_{t=1}^{T}\left(1+\gamma_{t}\right)^{w_{t}}-\prod_{t=1}^{T}\left(1-\gamma_{t}\right)^{w_{t}}}{\prod_{t=1}^{T}\left(1+\gamma_{t}\right)^{w_{t}}+\prod_{t=1}^{T}\left(1-\gamma_{t}\right)^{w_{t}}} \leq \max \gamma_{t}
$$

Let $\operatorname{HPFEWA}\left(\bar{h}_{1}, \bar{h}_{2}, \ldots, \bar{h}_{T}\right)=\bar{h}\left(\gamma_{i} \mid p_{1} p_{2} \cdots p_{T}\right), i=1,2, \ldots, \# \bar{h}$, where $\# \bar{h}=\# \bar{h}_{1} \times \# \bar{h}_{2} \times \cdots \times$ $\# \bar{h}_{T}, \bar{h}^{-}=\left(\min \gamma_{t} \mid p_{1} p_{2} \cdots p_{T}\right)$ and $\bar{h}^{+}=\left(\max \gamma_{t} \mid p_{1} p_{2} \cdots p_{T}\right)$; then, Equation (11) is transformed into the following form: $\min \gamma_{t} \leq \gamma_{i} \leq \max \gamma_{t}$ for all $i=1,2, \ldots, \# \bar{h}$. Thus, $s\left(\bar{h}^{-}\right)=\min \gamma_{t} p_{1} p_{2} \cdots p_{T} \leq$ $\sum_{i=1}^{\# \bar{h}} \gamma_{i} p_{1} p_{2} \cdots p_{T}=s(\bar{h})$ and $s(\bar{h})=\sum_{i=1}^{\# \bar{h}} \gamma_{i} p_{1} p_{2} \cdots p_{T} \leq \max \gamma_{t} p_{1} p_{2} \cdots p_{T}=s\left(\bar{h}^{+}\right)$.

If $s\left(\bar{h}^{-}\right)<s(\bar{h})$ and $s(\bar{h})<s\left(\bar{h}^{+}\right)$, then by Definition 3 , we have $\bar{h}^{-}<\operatorname{HPFEWA}\left(\bar{h}_{1}, \bar{h}_{2}, \ldots, \bar{h}_{T}\right)<$ $\bar{h}^{+}$. If $s(\bar{h})=s\left(\bar{h}^{+}\right)$, i.e., $\max \gamma_{t}=\sum_{i=1}^{\# \bar{h}} \gamma_{i}$, then $d(\bar{h})=\sum_{i=1}^{\# \bar{h}}\left(\gamma_{i}-s(\bar{h})\right)^{2} p_{1} p_{2} \cdots p_{T}=$ $\left(\max \gamma_{t}-s(\bar{h})\right)^{2} p_{1} p_{2} \cdots p_{T}=d\left(\bar{h}^{+}\right)$. In this case, in accordance with Definition 3, it follows that $\operatorname{HPFEWA}\left(\bar{h}_{1}, \bar{h}_{2}, \ldots, \bar{h}_{T}\right)=\bar{h}^{+}$. If $s(\bar{h})=s\left(\bar{h}^{-}\right)$, then similarly, we have $\operatorname{HPFEWA}\left(\bar{h}_{1}, \bar{h}_{2}, \ldots, \bar{h}_{T}\right)=\bar{h}^{-}$.

(2) Let $f(x)=\frac{1-x}{1+x}, x \in[0,1]$; then, $f(x)$ is a decreasing function. If $\gamma_{i}^{(t)} \leq \dot{\gamma}_{i}^{(t)}$ for each $i=1,2, \ldots, \#_{t}, t=1,2, \ldots, T$; then, $f\left(\gamma_{i}^{(t)}\right) \geq f\left(\dot{\gamma}_{i}^{(t)}\right)$, for each $i=1,2, \ldots, \#_{t}, t=1,2, \ldots, T$, i.e., $\frac{1-\gamma_{i}^{(t)}}{1+\gamma_{i}^{(t)}} \geq \frac{1-\dot{\gamma}_{i}^{(t)}}{1+\dot{\gamma}_{i}^{(t)}}$, for each $i=1,2, \ldots, \#_{t}, t=1,2, \ldots, T$. For any $\gamma_{i}^{(t)} \in \bar{h}_{t}(t=1,2, \ldots, T)$, since $w=\left(w_{1}, w_{2}, \ldots, w_{T}\right)^{T}$ is the weight vector of $\bar{h}_{t}(t=1,2, \ldots, T)$ such that $w_{t} \in[0,1], t=1,2, \ldots, T$ and $\sum_{t=1}^{T} w_{t}=1$, we have

$$
\left(\frac{1-\gamma_{i}^{(t)}}{1+\gamma_{i}^{(t)}}\right)^{w_{t}} \geq\left(\frac{1-\dot{\gamma}_{i}^{(t)}}{1+\dot{\gamma}_{i}^{(t)}}\right)^{w_{t}}, t=1,2, \ldots, T .
$$


Then,

$$
\begin{aligned}
& \prod_{t=1}^{T}\left(\frac{1-\gamma_{i}^{(t)}}{1+\gamma_{i}^{(t)}}\right)^{w_{t}} \geq \prod_{t=1}^{T}\left(\frac{1-\dot{\gamma}_{i}^{(t)}}{1+\dot{\gamma}_{i}^{(t)}}\right)^{w_{t}} \Leftrightarrow 1+\prod_{t=1}^{T}\left(\frac{1-\gamma_{i}^{(t)}}{1+\gamma_{i}^{(t)}}\right)^{w_{t}} \\
& \Leftrightarrow \frac{1+\prod_{t=1}^{T}\left(\frac{1-\dot{\gamma}_{i}^{(t)}}{1+\dot{\gamma}_{i}^{(t)}}\right)^{w_{t}}}{1+\prod_{t=1}^{T}\left(\frac{1-\gamma_{i}^{(t)}}{1+\gamma_{i}^{(t)}}\right)^{w_{t}}} \leq \frac{1}{1+\prod_{t=1}^{T}\left(\frac{1-\dot{\gamma}_{i}^{(t)}}{1+\dot{\gamma}_{i}^{(t)}}\right)^{w_{t}}} \\
& \Leftrightarrow \frac{2}{1+\prod_{t=1}^{T}\left(\frac{1-\gamma_{i}^{(t)}}{1+\gamma_{i}^{(t)}}\right)^{w_{t}}}-1 \leq \frac{2}{1+\prod_{t=1}^{T}\left(\frac{1-\dot{\gamma}_{i}^{(t)}}{1+\dot{\gamma}_{i}^{(t)}}\right)^{w_{t}}-1}
\end{aligned}
$$

i.e.,

$$
\frac{\prod_{t=1}^{T}\left(1+\gamma_{i}^{(t)}\right)^{w_{t}}-\prod_{t=1}^{T}\left(1-\gamma_{i}^{(t)}\right)^{w_{t}}}{\prod_{t=1}^{T}\left(1+\gamma_{i}^{(t)}\right)^{w_{t}}+\prod_{t=1}^{T}\left(1-\gamma_{i}^{(t)}\right)^{w_{t}}} \leq \frac{\prod_{t=1}^{T}\left(1+\dot{\gamma}_{i}^{(t)}\right)^{w_{t}}-\prod_{t=1}^{T}\left(1-\dot{\gamma}_{i}^{(t)}\right)^{w_{t}}}{\prod_{t=1}^{T}\left(1+\dot{\gamma}_{i}^{(t)}\right)^{w_{t}}+\prod_{t=1}^{T}\left(1-\dot{\gamma}_{i}^{(t)}\right)^{w_{t}}}
$$

Let $\operatorname{HPFEWA}\left(\bar{h}_{1}, \bar{h}_{2}, \ldots, \bar{h}_{T}\right)=\bar{h}\left(\gamma_{i} \mid p_{1} p_{2} \cdots p_{T}\right)$ and $\operatorname{HPFEWA}\left(\bar{h}_{1}^{*}, \bar{h}_{2}^{*}, \ldots, \bar{h}_{T}^{*}\right)=$ $\bar{h}^{*}\left(\dot{\gamma}_{i} \mid p_{1} p_{2} \cdots p_{T}\right)$, where $i=1,2, \ldots, \#$, and $\#=\#_{1} \times \#_{2} \times \cdots \times \#_{T}$ is the number of possible elements in $\bar{h}\left(\gamma_{i} \mid p_{1} p_{2} \cdots p_{T}\right)$ and $\bar{h}^{*}\left(\dot{\gamma}_{i} \mid p_{1} p_{2} \cdots p_{T}\right)$, respectively, then the Equation (12) is transformed into the form $\gamma_{i} \leq \dot{\gamma}_{i}\left(i=1,2, \ldots\right.$,\#). Thus, $s(\bar{h})=\sum_{i=1}^{\#} \gamma_{i} p_{1} p_{2} \cdots p_{T} \leq \sum_{i=1}^{\#} \dot{\gamma}_{i} p_{1} p_{2} \cdots p_{T}=s\left(\bar{h}^{*}\right)$.

If $s(\bar{h})<s\left(\bar{h}^{*}\right)$, then, according to Definition 3, we have $\operatorname{HPFEWA}\left(\bar{h}_{1}, \bar{h}_{2}, \ldots, \bar{h}_{T}\right)<$ $\operatorname{HPFEWA}\left(\bar{h}_{1}^{*}, \bar{h}_{2}^{*}, \ldots, \bar{h}_{T}^{*}\right)$. If $s(\bar{h})=s\left(\bar{h}^{*}\right)$, i.e., $\sum_{i=1}^{\#} \gamma_{i}=\sum_{i=1}^{\#} \dot{\gamma}_{i}$, then $d(\bar{h})=\sum_{i=1}^{\#}\left(\gamma_{i}-\right.$ $s(\bar{h}))^{2} p_{1} p_{2} \cdots p_{T}=\sum_{i=1}^{\#}\left(\dot{\gamma}_{i}-s\left(\bar{h}^{*}\right)\right)^{2} p_{1} p_{2} \cdots p_{T}=d\left(\bar{h}^{*}\right)$. In this case, based on Definition 3 , it follows that $\operatorname{HPFEWA}\left(\bar{h}_{1}, \bar{h}_{2}, \ldots, \bar{h}_{T}\right)=\operatorname{HPFEWA}\left(\bar{h}_{1}^{*}, \bar{h}_{2}^{*}, \ldots, \bar{h}_{T}^{*}\right)$.

However, the HPFEWA operator does not satisfy the idempotency. To illustrate this, we give the following example.

Example 3. Let $\bar{h}_{1}=\bar{h}_{2}=(0.3|0.5,0.7| 0.5)$, and $w=(0.2,0.8)^{T}$ is the weight vector $\bar{h}_{t}(t=1,2)$; then,

$$
\begin{aligned}
\operatorname{HPFEWA}\left(\bar{h}_{1}, \bar{h}_{2}\right) & =\cup_{\gamma_{1} \in \bar{h}_{1}, \gamma_{2} \in \bar{h}_{2}}\left\{\frac{\prod_{t=1}^{2}\left(1+\gamma_{t}\right)^{w_{t}}-\prod_{t=1}^{2}\left(1-\gamma_{t}\right)^{w_{t}}}{\prod_{t=1}^{2}\left(1+\gamma_{t}\right)^{w_{t}}+\prod_{t=1}^{2}\left(1-\gamma_{t}\right)^{w_{t}}} \mid p_{1} p_{2}\right\} \\
& =(0.3|0.25,0.398| 0.25,0.639|0.25,0.7| 0.25)
\end{aligned}
$$

and thus $\operatorname{HPFEWA}\left(\bar{h}_{1}, \bar{h}_{2}\right) \neq(0.3|0.5,0.7| 0.5)$.

Based on the HPFWG operator and Einstein operation, we developed the hesitant probabilistic fuzzy Einstein weighted geometric operator as follows:

Definition 9. Let $\bar{h}_{t}(t=1,2, \ldots, T)$ be a collection of HPFEs; then, the hesitant probabilistic fuzzy Einstein weighted geometric (HPFEWG) operator is a mapping $\left(H_{P}^{T} \rightarrow H_{P}\right)$ such that

$$
\operatorname{HPFEWG}\left(\bar{h}_{1}, \bar{h}_{2}, \ldots, \bar{h}_{T}\right)=\bar{h}_{1}^{\wedge_{\varepsilon} w_{1}} \otimes_{\varepsilon} \bar{h}_{2}^{\wedge_{\varepsilon} w_{2}} \otimes_{\varepsilon} \cdots \otimes_{\varepsilon} \bar{h}_{T}^{\wedge_{\varepsilon} w_{T}},
$$


where $w=\left(w_{1}, w_{2}, \ldots, w_{T}\right)^{T}$ is the weight vector of $\bar{h}_{t}(t=1,2, \ldots, T)$ with $w_{t} \in[0,1]$ and $\sum_{t=1}^{T} w_{t}=1$, and $p_{t}$ is the probability of $\gamma_{t}$ in HPFE $\bar{h}_{t}$. In particular, if $w=\left(\frac{1}{T}, \frac{1}{T}, \ldots, \frac{1}{T}\right)^{T}$, then the HPFEWG operator is reduced to the hesitant probabilistic fuzzy Einstein geometric (HPFEG) operator:

$$
\operatorname{HPFEG}\left(\bar{h}_{1}, \bar{h}_{2}, \ldots, \bar{h}_{T}\right)=\bar{h}_{1}^{\wedge_{\varepsilon} \frac{1}{T}} \otimes_{\varepsilon} \bar{h}_{2}^{\wedge_{\varepsilon} \frac{1}{T}} \otimes_{\varepsilon} \cdots \otimes_{\varepsilon} \bar{h}_{T}^{\wedge_{\varepsilon} \frac{1}{T}} .
$$

Theorem 6. Let $\bar{h}_{t}(t=1,2, \ldots, T)$ be a collection of HPFEs; then, their aggregated value obtained using the HPFEWG operator is also a HPFE and

$$
\operatorname{HPFEWG}\left(\bar{h}_{1}, \bar{h}_{2}, \ldots, \bar{h}_{T}\right)=\bigcup_{\gamma_{1} \in \bar{h}_{1}, \gamma_{2} \in \bar{h}_{2}, \ldots, \gamma_{T} \in \bar{h}_{T}}\left\{\frac{2 \prod_{t=1}^{T} \gamma_{t}^{w_{t}}}{\prod_{t=1}^{T}\left(2-\gamma_{t}\right)^{w_{t}}+\prod_{t=1}^{T} \gamma_{t}^{w_{t}}} \mid p_{1} p_{2} \cdots p_{T}\right\}
$$

where $w=\left(w_{1}, w_{2}, \ldots, w_{T}\right)^{T}$ is the weight vector of $\bar{h}_{t}(t=1,2, \ldots, T)$ with $w_{t} \in[0,1]$ and $\sum_{t=1}^{T} w_{t}=1$, and $p_{t}$ is the probability of $\gamma_{t}$ in HPFE $\bar{h}_{t}$.

Proof. We prove Equation (15) by mathematical induction on $T$. When $T=2$, since $\bar{h}_{1}^{\wedge_{\varepsilon} w_{1}}=$ $\cup_{\gamma_{1} \in \bar{h}_{1}}\left\{\frac{2 \gamma_{1}^{w_{1}}}{\left(2-\gamma_{1}\right)^{w_{1}}+\gamma_{1}^{w_{1}}} \mid p_{1}\right\}$ and $\bar{h}_{2}^{\wedge w_{2} w_{2}}=\cup_{\gamma_{2} \in \bar{h}_{2}}\left\{\frac{2 \gamma_{2}^{w_{2}}}{\left(2-\gamma_{2}\right)^{w_{2}}+\gamma_{2}^{w_{2}}} \mid p_{2}\right\}$, we have

$$
\begin{aligned}
\bar{h}_{1}^{\wedge_{\varepsilon} w_{1}} \otimes_{\varepsilon} \bar{h}_{2}^{\wedge_{\varepsilon} w_{2}} & =\bigcup_{\gamma_{1} \in \bar{h}_{1}, \gamma_{2} \in \bar{h}_{2}}\left\{\frac{\frac{2 \gamma_{1}^{w}}{\left(2-\gamma_{1}\right)^{w_{1}}+\gamma_{1}^{w_{1}}} \cdot \frac{2 \gamma_{2}^{z v_{2}}}{\left(2-\gamma_{2}\right)^{w_{2}}+\gamma_{2}^{w_{2}}}}{1+\left(1-\frac{2 \gamma_{1}^{w_{1}}}{\left(2-\gamma_{1}\right)^{2 w_{1}}+\gamma_{1}^{w w_{1}}}\right)\left(1-\frac{2 \gamma_{2}^{w_{2}}}{\left(2-\gamma_{2}\right)^{2 w_{2}}+\gamma_{2}^{w w_{2}}}\right)} p_{1} p_{2}\right\} \\
& =\bigcup_{\gamma_{1} \in \bar{h}_{1, \gamma_{2} \in \bar{h}_{2}}}\left\{\frac{2 \prod_{t=1}^{2} \gamma_{t}^{w w_{t}}}{\prod_{t=1}^{2}\left(2-\gamma_{t}\right)^{w_{t}}+\prod_{t=1}^{2} \gamma_{t}^{w_{t}}} \mid p_{1} p_{2}\right\} .
\end{aligned}
$$

Assume that Equation (15) holds for $T=k$, i.e.,

$$
\bar{h}_{1}^{\wedge_{\varepsilon} w_{1}} \otimes_{\varepsilon} \bar{h}_{2}^{\wedge_{\varepsilon} w_{2}} \otimes_{\varepsilon} \cdots \otimes_{\varepsilon} \bar{h}_{k}^{\wedge_{\varepsilon} w_{k}}=\bigcup_{\gamma_{1} \in \bar{h}_{1}, \gamma_{2} \in \bar{h}_{2}, \cdots, \gamma_{k} \in \bar{h}_{k}}\left\{\frac{2 \prod_{t=1}^{k} \gamma_{t}^{w_{t}}}{\prod_{t=1}^{k}\left(2-\gamma_{t}\right)^{w_{t}}+\prod_{t=1}^{k} \gamma_{t}^{w_{t}}} \mid p_{1} p_{2} \cdots p_{k}\right\} .
$$

In accordance with the Einstein operational laws of HPFEs for $T=k+1$, we have

$$
\begin{aligned}
& \bar{h}_{1}^{\wedge_{\varepsilon} w_{1}} \otimes_{\mathcal{E}} \bar{h}_{2}^{\wedge_{\varepsilon} w_{2}} \otimes_{\varepsilon} \cdots \otimes_{\mathcal{E}} \bar{h}_{k+1}^{\wedge_{\varepsilon} w_{k+1}}=\left(\bar{h}_{1}^{\wedge_{\varepsilon} w_{1}} \otimes_{\varepsilon} \bar{h}_{2}^{\wedge_{\varepsilon} w_{2}} \otimes_{\varepsilon} \cdots \otimes_{\varepsilon} \bar{h}_{k}^{\wedge_{\varepsilon} w_{k}}\right) \otimes_{\varepsilon} \bar{h}_{k+1}^{\wedge_{\varepsilon} w_{k+1}} \\
& =\bigcup_{\gamma_{1} \in \bar{h}_{1}, \gamma_{2} \in \bar{h}_{2, \ldots, \gamma_{k} \in \bar{h}_{k}}}\left\{\frac{2 \prod_{t=1}^{k} \gamma_{t}^{w_{t}}}{\prod_{t=1}^{k}\left(2-\gamma_{t}\right)^{w_{t}}+\prod_{t=1}^{k} \gamma_{t}^{w_{t}}} \mid p_{1} p_{2} \cdots p_{k}\right\} \\
& \otimes_{\varepsilon} \bigcup_{\gamma_{k+1} \in \bar{h}_{k+1}}\left\{\frac{2 \gamma_{k+1}^{w_{k+1}}}{\left(2-\gamma_{k+1}\right)^{w_{k+1}}+\gamma_{k+1}^{w_{k+1}}} \mid p_{k+1}\right\} \\
& =\bigcup_{\gamma_{1} \in \bar{h}_{1}, \gamma_{2} \in \bar{h}_{2}, \ldots, \gamma_{k} \in \bar{h}_{k}, \gamma_{k+1} \in \bar{h}_{k+1}}\left\{\frac{2 \prod_{t=1}^{k+1} \gamma_{t}^{w_{t}}}{\prod_{t=1}^{k+1}\left(2-\gamma_{t}\right)^{w_{t}}+\prod_{t=1}^{k+1} \gamma_{t}^{w_{t}}} \mid p_{1} p_{2} \cdots p_{k} p_{k+1}\right\} \text {, }
\end{aligned}
$$

i.e., Equation (15) holds for $T=k+1$. Then, Equation (15) holds for all $T$. Hence, we complete the proof of the theorem.

Based on Theorem 6, we have basic properties of the HPFEWG operator, as follows: 
Theorem 7. Let $\bar{h}_{t}\left(\gamma_{i}^{(t)} \mid p_{t}\right)(t=1,2, \ldots, T)$ be a collection of HPFEs, $w=\left(w_{1}, w_{2}, \ldots, w_{T}\right)^{T}$ be the weight vector of $\bar{h}_{t}(t=1,2, \ldots, T)$ such that $w_{t} \in[0,1]$ and $\sum_{t=1}^{T} w_{t}=1$, and $p_{t}$ be the corresponding probability of $\gamma_{i}^{(t)}$ in HPFE $\bar{h}_{t}$. Then, we have the following.

(1) (Boundary):

$$
\bar{h}^{-} \leq \operatorname{HPFEWG}\left(\bar{h}_{1}, \bar{h}_{2}, \ldots, \bar{h}_{T}\right) \leq \bar{h}^{+},
$$

where $\bar{h}^{-}=\left(\min _{1 \leq t \leq T} \min _{\gamma_{t} \in \bar{h}_{t}} \gamma_{t} \mid p_{1} p_{2} \cdots p_{T}\right)$ and $\bar{h}^{+}=\left(\max _{1 \leq t \leq T} \max _{\gamma_{t} \in \bar{h}_{t}} \gamma_{t} \mid p_{1} p_{2} \cdots p_{T}\right)$.

(2) (Monotonicity): Let $\bar{h}_{t}^{*}\left(\dot{\gamma}_{i}^{(t)} \mid p_{t}\right)(t=1,2, \ldots, T)$ be a collection of HPFEs with $\#_{t}=\# \bar{h}_{t}=\# \bar{h}_{t}^{*}$ for $t=1,2, \ldots, T, w=\left(w_{1}, w_{2}, \ldots, w_{T}\right)^{T}$ be the weight vector of $\bar{h}_{t}^{*}(t=1,2, \ldots, T)$ such that $w_{t} \in[0,1]$ and $\sum_{t=1}^{T} w_{t}=1$, and $p_{t}$ be the probability of $\dot{\gamma}_{i}^{(t)}$ in HPFE $\bar{h}_{t}^{*}$. If $\gamma_{i}^{(t)} \leq \dot{\gamma}_{i}^{(t)}$ for each $i=1,2, \ldots, \#_{t}$, $t=1,2, \ldots, T$, then

$$
\operatorname{HPFEWG}\left(\bar{h}_{1}, \bar{h}_{2}, \ldots, \bar{h}_{T}\right) \leq \operatorname{HPFEWG}\left(\bar{h}_{1}^{*}, \bar{h}_{2}^{*}, \ldots, \bar{h}_{T}^{*}\right) .
$$

Proof. (1) Let $g(x)=\frac{2-x}{x}, x \in(0,1]$; then, $g^{\prime}(x)=\frac{-2}{x^{2}}<0$, i.e., $g(x)$ is a decreasing function. Let $\max \gamma_{t}=\max _{1 \leq t \leq T} \max _{\gamma_{t} \in \bar{h}_{t}} \gamma_{t}$ and $\min \gamma_{t}=\min _{1 \leq t \leq T} \min _{\gamma_{t} \in \bar{h}_{t}} \gamma_{t}$. For any $\gamma_{t} \in \bar{h}_{t}(t=1,2, \ldots, T)$, since $\min _{\gamma_{t} \in \bar{h}_{t}} \gamma_{t} \leq \gamma_{t} \leq \max _{\gamma_{t} \in \bar{h}_{t}} \gamma_{t}$; then, $g\left(\max _{\gamma_{t} \in \bar{h}_{t}} \gamma_{t}\right) \leq g\left(\gamma_{t}\right) \leq g\left(\min _{\gamma_{t} \in \bar{h}_{t}} \gamma_{t}\right)$, and so

$$
\frac{2-\max \gamma_{t}}{\max \gamma_{t}} \leq \frac{2-\max _{\gamma_{t} \in \bar{h}_{t}} \gamma_{t}}{\max _{\gamma_{t} \in \bar{h}_{t}} \gamma_{t}} \leq \frac{2-\gamma_{t}}{\gamma_{t}} \leq \frac{2-\min _{\gamma_{t} \in \bar{h}_{t}} \gamma_{t}}{\min _{\gamma_{t} \in \bar{h}_{t}} \gamma_{t}} \leq \frac{2-\min \gamma_{t}}{\min \gamma_{t}}
$$

Since $w=\left(w_{1}, w_{2}, \ldots, w_{T}\right)^{T}$ is the weight vector of $\bar{h}_{t}(t=1,2, \ldots, T)$ with $w_{t} \in[0,1]$ and $\sum_{t=1}^{T} w_{t}=1$, we have

$$
\prod_{t=1}^{T}\left(\frac{2-\max \gamma_{t}}{\max \gamma_{t}}\right)^{w_{t}} \leq \prod_{t=1}^{T}\left(\frac{2-\gamma_{t}}{\gamma_{t}}\right)^{w_{t}} \leq \prod_{t=1}^{T}\left(\frac{2-\min \gamma_{t}}{\min \gamma_{t}}\right)^{w_{t}}
$$

Since $\prod_{t=1}^{T}\left(\frac{2-\max \gamma_{t}}{\max \gamma_{t}}\right)^{w_{t}}=\left(\frac{2-\max \gamma_{t}}{\max \gamma_{t}}\right)^{\sum_{t=1}^{T} w_{t}}=\frac{2-\max \gamma_{t}}{\max \gamma_{t}}$ and $\prod_{t=1}^{T}\left(\frac{2-\min \gamma_{t}}{\min \gamma_{t}}\right)^{w_{t}}=$ $\left(\frac{2-\min \gamma_{t}}{\min \gamma_{t}}\right)^{\sum_{t=1}^{T} w_{t}}=\frac{2-\min \gamma_{t}}{\min \gamma_{t}}$, we obtain

$$
\begin{aligned}
& \frac{2-\max \gamma_{t}}{\max \gamma_{t}} \leq \prod_{t=1}^{T}\left(\frac{2-\gamma_{t}}{\gamma_{t}}\right)^{w_{t}} \leq \frac{2-\min \gamma_{t}}{\min \gamma_{t}} \Leftrightarrow \frac{2}{\max \gamma_{t}} \leq 1+\prod_{t=1}^{T}\left(\frac{2-\gamma_{t}}{\gamma_{t}}\right)^{w_{t}} \leq \frac{2}{\min \gamma_{t}} \\
& \Leftrightarrow \frac{\min \gamma_{t}}{2} \leq \frac{1}{1+\prod_{t=1}^{T}\left(\frac{2-\gamma_{t}}{\gamma_{t}}\right)^{w_{t}}} \leq \frac{\max \gamma_{t}}{2} \\
& \Leftrightarrow \min \gamma_{t} \leq \frac{2}{1+\prod_{t=1}^{T}\left(\frac{2-\gamma_{t}}{\gamma_{t}}\right)^{w_{t}}} \leq \max \gamma_{t},
\end{aligned}
$$

i.e.,

$$
\min \gamma_{t} \leq \frac{2 \prod_{t=1}^{T} \gamma_{t}^{w_{t}}}{\prod_{t=1}^{T}\left(2-\gamma_{t}\right)^{w_{t}}+\prod_{t=1}^{T} \gamma_{t}^{w_{t}}} \leq \max \gamma_{t}
$$

Let HPFEWG $\left(\bar{h}_{1}, \bar{h}_{2}, \ldots, \bar{h}_{T}\right)=\bar{h}\left(\gamma_{i} \mid p_{1} p_{2} \cdots p_{T}\right), i=1,2, \ldots, \# \bar{h}$, where $\# \bar{h}=\# \bar{h}_{1} \times \# \bar{h}_{2} \times$ $\cdots \times \# \bar{h}_{T}, \bar{h}^{-}=\left(\min \gamma_{t} \mid p_{1} p_{2} \cdots p_{T}\right)$ and $\bar{h}^{+}=\left(\max \gamma_{t} \mid p_{1} p_{2} \cdots p_{T}\right)$. Then, Equation (18) is transformed into the following forms: $\min \gamma_{t} \leq \gamma_{i} \leq \max \gamma_{t}$ for all $i=1,2, \ldots, \# \bar{h}$. Thus, $s\left(\bar{h}^{-}\right)=\min \gamma_{t} p_{1} p_{2} \cdots p_{T} \leq \sum_{i=1}^{\# \bar{h}} \gamma_{i} p_{1} p_{2} \cdots p_{T}=s(\bar{h})$ and $s(\bar{h})=\sum_{i=1}^{\# \bar{h}_{i}} \gamma_{i} p_{1} p_{2} \cdots p_{T} \leq$ $\max \gamma_{t} p_{1} p_{2} \cdots p_{T}=s\left(\bar{h}^{+}\right)$. If $s\left(\bar{h}^{-}\right)<s(\bar{h})$ and $s(\bar{h})<s\left(\bar{h}^{+}\right)$. Then, based on Definition 3, we have $\bar{h}^{-}<\operatorname{HPFEWG}\left(\bar{h}_{1}, \bar{h}_{2}, \ldots, \bar{h}_{T}\right)<\bar{h}^{+}$. If $s(\bar{h})=s\left(\bar{h}^{+}\right)$, i.e., $\max \gamma_{t}=\sum_{i=1}^{\# \bar{h}} \gamma_{i}$, then 
$d(\bar{h})=\sum_{i=1}^{\# \bar{h}}\left(\gamma_{i}-s(\bar{h})\right)^{2} p_{1} p_{2} \cdots p_{T}=\left(\max \gamma_{t}-s(\bar{h})\right)^{2} p_{1} p_{2} \cdots p_{T}=d\left(\bar{h}^{+}\right)$. In this case, based on Definition 3 , it follows that HPFEWG $\left(\bar{h}_{1}, \bar{h}_{2}, \ldots, \bar{h}_{T}\right)=\bar{h}^{+}$. If $s(\bar{h})=s\left(\bar{h}^{-}\right)$. Then, similarly, we have $\operatorname{HPFEWG}\left(\bar{h}_{1}, \bar{h}_{2}, \ldots, \bar{h}_{T}\right)=\bar{h}^{-}$.

(2) Let $g(x)=\frac{2-x}{x}, x \in(0,1]$; then, $g(x)$ is a decreasing function. If $\gamma_{i}^{(t)} \leq \dot{\gamma}_{i}^{(t)}$ for each $i=1,2, \ldots, \#_{t}, t=1,2, \ldots, T$, then $g\left(\gamma_{i}^{(t)}\right) \geq g\left(\dot{\gamma}_{i}^{(t)}\right)$, for each $i=1,2, \ldots, \#_{t}, t=1,2, \ldots, T$, i.e., $\frac{2-\gamma_{i}^{(t)}}{\gamma_{i}^{(t)}} \geq \frac{2-\dot{\gamma}_{i}^{(t)}}{\dot{\gamma}_{i}^{(t)}}$, for each $i=1,2, \ldots, \#_{t}, t=1,2, \ldots, T$. For any $\gamma_{i}^{(t)} \in \bar{h}_{t}(t=1,2, \ldots, T)$, since $w=\left(w_{1}, w_{2}, \ldots, w_{T}\right)^{T}$ is the weight vector of $\bar{h}_{t}(t=1,2, \ldots, T)$ such that $w_{t} \in[0,1], t=1,2, \ldots, T$ and $\sum_{t=1}^{T} w_{t}=1$, we have

$$
\left(\frac{2-\gamma_{i}^{(t)}}{\gamma_{i}^{(t)}}\right)^{w_{t}} \geq\left(\frac{2-\dot{\gamma}_{i}^{(t)}}{\dot{\gamma}_{i}^{(t)}}\right)^{w_{t}}, i=1,2, \ldots, \#_{t}, t=1,2, \ldots, T .
$$

Then,

$$
\begin{aligned}
\prod_{t=1}^{T}\left(\frac{2-\gamma_{i}^{(t)}}{\gamma_{i}^{(t)}}\right)^{w_{t}} \geq \prod_{t=1}^{T}\left(\frac{2-\dot{\gamma}_{i}^{(t)}}{\dot{\gamma}_{i}^{(t)}}\right)^{w_{t}} & \Leftrightarrow 1+\prod_{t=1}^{T}\left(\frac{2-\gamma_{i}^{(t)}}{\gamma_{i}^{(t)}}\right)^{w_{t}} \geq 1+\prod_{t=1}^{T}\left(\frac{2-\dot{\gamma}_{i}^{(t)}}{\dot{\gamma}_{i}^{(t)}}\right)^{w_{t}} \\
& \Leftrightarrow \frac{1}{1+\prod_{t=1}^{T}\left(\frac{2-\gamma_{i}^{(t)}}{\gamma_{i}^{(t)}}\right)^{w_{t}}} \leq \frac{1}{1+\prod_{t=1}^{T}\left(\frac{2-\dot{\gamma}_{i}^{(t)}}{\dot{\gamma}_{i}^{(t)}}\right)^{w_{t}}} \\
& \Leftrightarrow \frac{2}{1+\prod_{t=1}^{T}\left(\frac{2-\gamma_{i}^{(t)}}{\gamma_{i}^{(t)}}\right)^{w_{t}}}-1 \leq \frac{2}{1+\prod_{t=1}^{T}\left(\frac{2-\dot{\gamma}_{i}^{(t)}}{\dot{\gamma}_{i}^{(t)}}\right)^{w_{t}}}-1,
\end{aligned}
$$

i.e.,

$$
\frac{2 \prod_{t=1}^{T}\left(\gamma_{i}^{(t)}\right)^{w_{t}}}{\prod_{t=1}^{T}\left(2-\gamma_{i}^{(t)}\right)^{w_{t}}+\prod_{t=1}^{T}\left(\gamma_{i}^{(t)}\right)^{w_{t}}} \leq \frac{2 \prod_{t=1}^{T}\left(\gamma_{i}^{(t)}\right)^{w_{t}}}{\prod_{t=1}^{T}\left(2-\gamma_{i}^{(t)}\right)^{w_{t}}+\prod_{t=1}^{T}\left(\gamma_{i}^{(t)}\right)^{w_{t}}}
$$

Let HPFEWG $\left(\bar{h}_{1}, \bar{h}_{2}, \ldots, \bar{h}_{T}\right)=\bar{h}\left(\gamma_{i} \mid p_{1} p_{2} \cdots p_{T}\right)$ and $\operatorname{HPFEWG}\left(\bar{h}_{1}^{*}, \bar{h}_{2}^{*}, \ldots, \bar{h}_{T}^{*}\right)=$ $\bar{h}^{*}\left(\dot{\gamma}_{i} \mid p_{1} p_{2} \cdots p_{T}\right)$, where $i=1,2, \ldots, \#$, and $\#=\#_{1} \times \#_{2} \times \cdots \times \#_{T}$ is the number of possible elements in $\bar{h}\left(\gamma_{i} \mid p_{1} p_{2} \cdots p_{T}\right)$ and $\bar{h}^{*}\left(\dot{\gamma}_{i} \mid p_{1} p_{2} \cdots p_{T}\right)$, respectively. Then, the Equation (19) is transformed into the form $\gamma_{i} \leq \dot{\gamma}_{i}\left(i=1,2, \ldots\right.$,\#). Thus, $s(\bar{h})=\sum_{i=1}^{\#} \gamma_{i} p_{1} p_{2} \cdots p_{T} \leq \sum_{i=1}^{\#} \dot{\gamma}_{i} p_{1} p_{2} \cdots p_{T}=s\left(\bar{h}^{*}\right)$. If $s(\bar{h})<s\left(\bar{h}^{*}\right)$, then based on Definition 3, HPFEWG $\left(\bar{h}_{1}, \bar{h}_{2}, \ldots, \bar{h}_{T}\right)<\operatorname{HPFEWG}\left(\bar{h}_{1}^{*}, \bar{h}_{2}^{*}, \ldots, \bar{h}_{T}^{*}\right)$. If $s(\bar{h})=s\left(\bar{h}^{*}\right)$, i.e., $\sum_{i=1}^{\#} \gamma_{i}=\sum_{i=1}^{\#} \dot{\gamma}_{i}$, then $d(\bar{h})=\sum_{i=1}^{\#}\left(\gamma_{i}-s(\bar{h})\right)^{2} p_{1} p_{2} \cdots p_{T}=$ $\sum_{i=1}^{\#}\left(\dot{\gamma}_{i}-s\left(\bar{h}^{*}\right)\right)^{2} p_{1} p_{2} \cdots p_{T}=d\left(\bar{h}^{*}\right)$. In this case, based on Definition 3, it follows that $\operatorname{HPFEWG}\left(\bar{h}_{1}, \bar{h}_{2}, \ldots, \bar{h}_{T}\right)=\operatorname{HPFEWG}\left(\bar{h}_{1}^{*}, \bar{h}_{2}^{*}, \ldots, \bar{h}_{T}^{*}\right)$.

If all probabilities of values in each HPFE are equal, i.e., $p_{1}=p_{2}=\cdots=p_{\# \bar{h}_{t}}(t=1,2, \ldots, T)$, then the HPFE is reduced to the HFE. In this case, the score function of the HPFEWA (resp. HPFEWG) operator is consistent with that of the HFEWA (resp. HFEWG) operator [21]. So, we can conclude that the HPFEWA (resp. HPFEWG) operator is reduced to the HFEWA (resp. HFEWG) operator [21]. In order to analyze the relationship between the HPFEWA (resp. HPFEWG) operator and the HPFWA (resp. HPFWG) operator [27], we introduce the following lemma.

Lemma 1. [41,42] Let $x_{i}>0, w_{i}>0, i=1,2, \ldots, N$, and $\sum_{i=1}^{N} w_{i}=1$, then $\prod_{i=1}^{N} x_{i}^{w_{i}} \leq \sum_{i=1}^{N} w_{i} x_{i}$, with equality if and only if $x_{1}=x_{2}=\cdots=x_{N}$.

Theorem 8. If $\bar{h}_{t}(t=1,2, \ldots, T)$ are a collection of HPFEs and $w=\left(w_{1}, w_{2}, \ldots, w_{T}\right)^{T}$ is the weight vector of $\bar{h}_{t}$, with $w_{t} \in[0,1]$ and $\sum_{t=1}^{T} w_{t}=1$, and $p_{t}$ is the probability of $\gamma_{t}$ in HPFE $\bar{h}_{t}$, then

(1) $\operatorname{HPFEWA}\left(\bar{h}_{1}, \bar{h}_{2}, \ldots, \bar{h}_{T}\right) \leq \operatorname{HPFWA}\left(\bar{h}_{1}, \bar{h}_{2}, \ldots, \bar{h}_{T}\right)$; 
(2) $\operatorname{HPFEWG}\left(\bar{h}_{1}, \bar{h}_{2}, \ldots, \bar{h}_{T}\right) \geq \operatorname{HPFWG}\left(\bar{h}_{1}, \bar{h}_{2}, \ldots, \bar{h}_{T}\right)$.

Proof. (1) For any $\gamma_{t} \in \bar{h}_{t}(t=1,2, \ldots, T)$, based on Lemma 1, we obtain the inequality $\prod_{t=1}^{T}(1+$ $\left.\gamma_{t}\right)^{w_{t}}+\prod_{t=1}^{T}\left(1-\gamma_{t}\right)^{w_{t}} \leq \sum_{t=1}^{T} w_{t}\left(1+\gamma_{t}\right)+\sum_{t=1}^{T} w_{t}\left(1-\gamma_{t}\right)=2$, and then

$$
\frac{\prod_{t=1}^{T}\left(1+\gamma_{t}\right)^{w_{t}}-\prod_{t=1}^{T}\left(1-\gamma_{t}\right)^{w_{t}}}{\prod_{t=1}^{T}\left(1+\gamma_{t}\right)^{w_{t}}+\prod_{t=1}^{T}\left(1-\gamma_{t}\right)^{w_{t}}}=1-\frac{2 \prod_{t=1}^{T}\left(1-\gamma_{t}\right)^{w_{t}}}{\prod_{t=1}^{T}\left(1+\gamma_{t}\right)^{w_{t}}+\prod_{t=1}^{T}\left(1-\gamma_{t}\right)^{w_{t}}} \leq 1-\prod_{t=1}^{T}\left(1-\gamma_{t}\right)^{w_{t}} .
$$

Hence, we can obtain the inequality

$$
\begin{array}{r}
\bigcup_{\gamma_{1} \in \bar{h}_{1}, \gamma_{2} \in \bar{h}_{2}, \ldots, \gamma_{T} \in \bar{h}_{T}}\left\{\frac{\prod_{t=1}^{T}\left(1+\gamma_{t}\right)^{w_{t}}-\prod_{t=1}^{T}\left(1-\gamma_{t}\right)^{w_{t}}}{\prod_{t=1}^{T}\left(1+\gamma_{t}\right)^{w_{t}}+\prod_{t=1}^{T}\left(1-\gamma_{t}\right)^{w_{t}}}\right\} \leq \\
\bigcup_{\gamma_{1} \in \bar{h}_{1}, \gamma_{2} \in \bar{h}_{2, \ldots, \gamma_{T} \in \bar{h}_{T}}\left\{1-\prod_{t=1}^{T}\left(1-\gamma_{t}\right)^{w_{t}}\right\} .}
\end{array}
$$

Let HPFEWA $\left(\bar{h}_{1}, \bar{h}_{2}, \ldots, \bar{h}_{T}\right)=\bar{h}\left(\gamma_{i} \mid p_{i}\right)$ and $\operatorname{HPFWA}\left(\bar{h}_{1}, \bar{h}_{2}, \ldots, \bar{h}_{T}\right)=\bar{h}^{*}\left(\gamma_{i}^{*} \mid p_{i}\right), i=1,2, \ldots \#$, where $\#=\# \bar{h}=\# \bar{h}^{*}$ is the number of possible elements in $\bar{h}\left(\gamma_{i} \mid p_{i}\right)$ and $\bar{h}^{*}\left(\dot{\gamma}_{i} \mid p_{i}\right)$, respectively. Then, Equation (21) is transformed into the form $\gamma_{i} \leq \gamma_{i}^{*}(i=1,2, \ldots, \#)$. According to $s(\bar{h})=\sum_{i=1}^{\# \bar{h}} \gamma_{i} p_{i}$, we have $\operatorname{HPFEWA}\left(\bar{h}_{1}, \bar{h}_{2}, \ldots, \bar{h}_{T}\right) \leq \operatorname{HPFWA}\left(\bar{h}_{1}, \bar{h}_{2}, \ldots, \bar{h}_{T}\right)$.

(2) For any $\gamma_{t} \in \bar{h}_{t}(t=1,2, \ldots, T)$, bsed on Lemma 1, we have $\prod_{t=1}^{T}\left(2-\gamma_{t}\right)^{w_{t}}+\prod_{t=1}^{T} \gamma_{t}^{w_{t}} \leq$ $\sum_{t=1}^{T} w_{t}\left(2-\gamma_{t}\right)+\sum_{t=1}^{T} w_{t} \gamma_{t}=2$, and then

$$
\frac{2 \prod_{t=1}^{T} \gamma_{t}^{w_{t}}}{\prod_{t=1}^{T}\left(2-\gamma_{t}\right)^{w_{t}}+\prod_{t=1}^{T} \gamma_{t}^{w_{t}}} \geq \prod_{t=1}^{T} \gamma_{t}^{w_{t}} .
$$

Hence, similarly to (1), we have $\operatorname{HPFEWG}\left(\bar{h}_{1}, \bar{h}_{2}, \ldots, \bar{h}_{T}\right) \geq \operatorname{HPFWG}\left(\bar{h}_{1}, \bar{h}_{2}, \ldots, \bar{h}_{T}\right)$.

Example 4. Let $\bar{h}_{1}=(0.5|0.5,0.6| 0.5)$ and $\bar{h}_{2}=(0.1|0.2,0.3| 0.3,0.4 \mid 0.5)$ be two HPFEs and $w=(0.6,0.4)^{T}$ be the weight vector of them. Then, based on Equation (8), the aggregated value from the HPFEWA operator is

$$
\begin{aligned}
\operatorname{HPFEWA}\left(\bar{h}_{1}, \bar{h}_{2}\right) & =\left(w_{1} \cdot \varepsilon \bar{h}_{1}\right) \oplus_{\varepsilon}\left(w_{2} \cdot \varepsilon \bar{h}_{2}\right) \\
& =\bigcup_{\gamma_{1} \in \bar{h}_{1}, \gamma_{2} \in \bar{h}_{2}}\left\{\frac{\prod_{t=1}^{2}\left(1+\gamma_{t}\right)^{w_{t}}-\prod_{t=1}^{2}\left(1-\gamma_{t}\right)^{w_{t}}}{\prod_{t=1}^{2}\left(1+\gamma_{t}\right)^{w_{t}}+\prod_{t=1}^{2}\left(1-\gamma_{t}\right)^{w_{t}}} \mid p_{1} p_{2}\right\} \\
& =\{0.3537|0.1,0.4247| 0.15,0.4614|0.25,0.4268| 0.1,0.4928|0.15,0.5265| 0.25\} .
\end{aligned}
$$

If we use the HPFWA operator (Equation (2)) to aggregate two HPFEs, then we have

$$
\begin{aligned}
\operatorname{HPFWA}\left(\bar{h}_{1}, \bar{h}_{2}\right) & =\left(w_{1} \bar{h}_{1}\right) \oplus\left(w_{2} \bar{h}_{2}\right) \\
& =\bigcup_{\gamma_{1} \in \bar{h}_{1}, \gamma_{2} \in \bar{h}_{2}}\left\{1-\prod_{t=1}^{2}\left(1-\gamma_{t}\right)^{w_{t}} \mid p_{1} p_{2}\right\} \\
& =\{0.3675|0.1,0.4280| 0.15,0.4622|0.25,0.4467| 0.1,0.4996|0.15,0.5296| 0.25\} .
\end{aligned}
$$

Then, $s\left(\operatorname{HPFEWA}\left(\bar{h}_{1}, \bar{h}_{2}\right)\right)=0.4627$ and $s\left(\operatorname{HPFWA}\left(\bar{h}_{1}, \bar{h}_{2}\right)\right)=0.4685$, and thus, $\operatorname{HPFEWA}\left(\bar{h}_{1}, \bar{h}_{2}\right)<\operatorname{HPFWA}\left(\bar{h}_{1}, \bar{h}_{2}\right)$. 
On the other hand, based on Equation (15), the aggregated value by HPFEWG operator is

$$
\begin{aligned}
\operatorname{HPFEWG}\left(\bar{h}_{1}, \bar{h}_{2}\right) & =\bar{h}_{1}^{\wedge_{\varepsilon} w_{1}} \otimes_{\varepsilon} \bar{h}_{2}^{\wedge_{\varepsilon} w_{2}} \\
& =\bigcup_{\gamma_{1} \in \bar{h}_{1}, \gamma_{2} \in \bar{h}_{2}}\left\{\frac{2 \prod_{t=1}^{2} \gamma_{t}^{w_{t}}}{\prod_{t=1}^{2}\left(2-\gamma_{t}\right)^{w_{t}}+\prod_{t=1}^{2} \gamma_{t}^{w_{t}}} \mid p_{1} p_{2}\right\} \\
& =\{0.2748|0.1,0.4108| 0.15,0.4581|0.25,0.3126| 0.1,0.4622|0.15,0.5135| 0.25\} .
\end{aligned}
$$

If we use the HPFWG operator (Equation (3)) to aggregate two HPFEs, then we get

$$
\begin{aligned}
\operatorname{HPFWG}\left(\bar{h}_{1}, \bar{h}_{2}\right) & =\left(\bar{h}_{1}\right)^{w_{1}} \otimes\left(\bar{h}_{2}\right)^{w_{2}} \\
& =\bigcup_{\gamma_{1} \in \bar{h}_{1}, \gamma_{2} \in \bar{h}_{2}}\left\{\prod_{t=1}^{2}\left(\gamma_{t}\right)^{w_{t}} \mid p_{1} p_{2}\right\} \\
& =\{0.2627|0.1,0.4076| 0.15,0.4573|0.25,0.2930| 0.1,0.4547|0.15,0.5102| 0.25\} .
\end{aligned}
$$

It is clear that $\operatorname{HPFEWG}\left(\bar{h}_{1}, \bar{h}_{2}\right)>\operatorname{HPFWG}\left(\bar{h}_{1}, \bar{h}_{2}\right)$.

Theorem 9. If $\bar{h}_{t}(t=1,2, \ldots, T)$ are a collection of HPFEs, $w=\left(w_{1}, w_{2}, \ldots, w_{T}\right)^{T}$ is the weight vector of $\bar{h}_{t}$ with $w_{t} \in[0,1]$ and $\sum_{t=1}^{T} w_{t}=1$, and $p_{t}$ is the probability of $\gamma_{t}$ in HPFE $\bar{h}_{t}$. Then,

(1) $\operatorname{HPFEWA}\left(\left(\bar{h}_{1}\right)^{c},\left(\bar{h}_{2}\right)^{c}, \ldots,\left(\bar{h}_{T}\right)^{c}\right)=\left(\operatorname{HPFEWG}\left(\bar{h}_{1}, \bar{h}_{2}, \ldots, \bar{h}_{T}\right)\right)^{c}$;

(2) $\operatorname{HPFEWG}\left(\left(\bar{h}_{1}\right)^{c},\left(\bar{h}_{2}\right)^{c}, \ldots,\left(\bar{h}_{T}\right)^{c}\right)=\left(\operatorname{HPFEWA}\left(\bar{h}_{1}, \bar{h}_{2}, \ldots, \bar{h}_{T}\right)\right)^{c}$.

Proof. Since (2) is similar (1), we only prove (1).

$$
\begin{aligned}
& \operatorname{HPFEWA}\left(\left(\bar{h}_{1}\right)^{\mathcal{C}},\left(\bar{h}_{2}\right)^{\mathcal{c}}, \ldots,\left(\bar{h}_{T}\right)^{\mathcal{C}}\right) \\
& =\bigcup_{\gamma_{1} \in \bar{h}_{1}, \gamma_{2} \in \bar{h}_{2}, \ldots, \gamma_{T} \in \bar{h}_{T}}\left\{\frac{\prod_{t=1}^{T}\left(1+\left(1-\gamma_{t}\right)\right)^{w_{t}}-\prod_{t=1}^{T}\left(1-\left(1-\gamma_{t}\right)\right)^{w_{t}}}{\prod_{t=1}^{T}\left(1+\left(1-\gamma_{t}\right)\right)^{w_{t}}+\prod_{t=1}^{T}\left(1-\left(1-\gamma_{t}\right)\right)^{w_{t}}} \mid p_{1} p_{2} \cdots p_{T}\right\} \\
& =\bigcup_{\gamma_{1} \in \bar{h}_{1}, \gamma_{2} \in \bar{h}_{2}, \ldots, \gamma_{T} \in \bar{h}_{T}}\left\{1-\frac{2 \prod_{t=1}^{T} \gamma_{t}^{w_{t}}}{\prod_{t=1}^{T}\left(2-\gamma_{t}\right)^{w_{t}}+\prod_{t=1}^{T} \gamma_{t}^{w_{t}}} \mid p_{1} p_{2} \cdots p_{T}\right\} \\
& =\left(\operatorname{HPFEWG}\left(\bar{h}_{1}, \bar{h}_{2}, \ldots, \bar{h}_{T}\right)\right)^{c} \text {. }
\end{aligned}
$$

Theorem 8 shows that (1) the values aggregated by the HPFEWA operator are not larger than those obtained by the HPFWA operator. That is to say, the HPFEWA operator reflects the decision maker's pessimistic attitude rather than the HPFWA operator in the aggregation process; and (2) the values aggregated by the HPFWG operator are not larger than those obtained by the HPFEWG operator. Thus, the HPFEWG operator reflects the decision maker's optimistic attitude rather than the HPFWG operator in the aggregation process. Moreover, we developed the following ordered weighted operators based on the HPFOWA operator [27] and the HPFOWG operator [27] to aggregate the HPFEs.

Let $\bar{h}_{t}(t=1,2, \ldots, T)$ be a collection of HPFEs, $\bar{h}_{\sigma(t)}$ be the $t$ th largest of $\bar{h}_{t}(t=1,2, \ldots, T)$, and $p_{\sigma(t)}$ be the probability of $\gamma_{\sigma(t)}$ in the HPFE $\bar{h}_{\sigma(t)}$; then, we have the following two aggregation operators, which are based on the mapping $H_{P}^{T} \rightarrow H_{P}$ with an associated vector $\omega=\left(\omega_{1}, \omega_{2}, \ldots, \omega_{T}\right)^{T}$, such that $\omega_{t} \in[0,1]$ and $\sum_{t=1}^{T} \omega_{t}=1$ :

(1) The hesitant probabilistic fuzzy Einstein ordered weighted averaging (HPFEOWA) operator is

$$
\begin{aligned}
& \operatorname{HPFEOWA}\left(\bar{h}_{1}, \bar{h}_{2}, \ldots, \bar{h}_{T}\right)=\left(\omega_{1} \cdot{ }_{\varepsilon} \bar{h}_{\sigma(1)}\right) \oplus_{\varepsilon}\left(\omega_{2} \cdot{ }_{\varepsilon} \bar{h}_{\sigma(2)}\right) \oplus_{\varepsilon} \cdots \oplus_{\varepsilon}\left(\omega_{T} \cdot \bar{h}_{\sigma(T)}\right) \\
& \quad=\bigcup_{\gamma_{\sigma(1)} \in \bar{h}_{\sigma(1)}, \gamma_{\sigma(2)} \in \bar{h}_{\sigma(2)}, \ldots, \gamma_{\sigma(T)} \in \bar{h}_{\sigma(T)}}\left\{\frac{\prod_{t=1}^{T}\left(1+\gamma_{\sigma(t)}\right)^{\omega_{t}}-\prod_{t=1}^{T}\left(1-\gamma_{\sigma(t)}\right)^{\omega_{t}}}{\prod_{t=1}^{T}\left(1+\gamma_{\sigma(t)}\right)^{\omega_{t}}+\prod_{t=1}^{T}\left(1-\gamma_{\sigma(t)}\right)^{\omega_{t}}} \mid p_{\sigma(1)} p_{\sigma(2)} \cdots p_{\sigma(T)}\right\} .
\end{aligned}
$$


(2) The hesitant probabilistic fuzzy Einstein ordered weighted geometric (HPFEOWG) operator is

$$
\begin{aligned}
& \operatorname{HPFEOWG}\left(\bar{h}_{1}, \bar{h}_{2}, \ldots, \bar{h}_{T}\right)=\left(\bar{h}_{\sigma(1)}^{\wedge_{\varepsilon} \omega_{1}}\right) \otimes_{\mathcal{E}}\left(\bar{h}_{\sigma(2)}^{\wedge_{\varepsilon} \omega_{2}}\right) \otimes_{\mathcal{E}} \cdots \otimes_{\mathcal{E}}\left(\bar{h}_{\sigma(T)}^{\wedge_{\varepsilon} \omega_{T}}\right) \\
& \quad=\bigcup_{\gamma_{\sigma(1)} \in \bar{h}_{\sigma(1)}, \gamma_{\sigma(2)} \in \bar{h}_{\sigma(2)}, \ldots, \gamma_{\sigma(T)} \in \bar{h}_{\sigma(T)}}\left\{\frac{2 \prod_{t=1}^{T} \gamma_{\sigma(t)}^{\omega_{t}}}{\prod_{t=1}^{T}\left(2-\gamma_{\sigma(t)}\right)_{t}+\prod_{t=1}^{T} \gamma_{\sigma(t)}^{\omega_{t}}} \mid p_{\sigma(1)} p_{\sigma(2)} \cdots p_{\sigma(T)}\right\} .
\end{aligned}
$$

Example 5. Let $\bar{h}_{1}=(0.5|0.5,0.6| 0.5)$ and $\bar{h}_{2}=(0.1|0.2,0.3| 0.3,0.4 \mid 0.5)$ be two HPFEs, and suppose that the associated aggregated vector is $\omega=(0.55,0.45)^{T}$. Based on Definition 3 , the score values of $\bar{h}_{1}$ and $\bar{h}_{2}$ are $s\left(\bar{h}_{1}\right)=0.55$ and $s\left(\bar{h}_{2}\right)=0.31$. Since $s\left(\bar{h}_{1}\right)>s\left(\bar{h}_{2}\right)$; then,

$$
\bar{h}_{\sigma(1)}=\bar{h}_{1}=(0.5|0.5,0.6| 0.5), \bar{h}_{\sigma(2)}=\bar{h}_{2}=(0.1|0.2,0.3| 0.3,0.4 \mid 0.5) .
$$

Based on Equation (21), the aggregated values by the HPFEOWA operator are

$$
\begin{aligned}
\operatorname{HPFEOWA}\left(\bar{h}_{1}, \bar{h}_{2}\right) & =\left(\omega_{1} \cdot \bar{h}_{\sigma(1)}\right) \oplus_{\varepsilon}\left(\omega_{2} \cdot{ }_{\varepsilon} \bar{h}_{\sigma(2)}\right) \\
& =\{0.3340|0.1,0.4023| 0.1,0.4148|0.15,0.4564| 0.25,0.4781|0.15,0.5167| 0.25\} .
\end{aligned}
$$

On the other hand, based on Equation (22), the aggregated values by the HPFEOWG operator are

$$
\begin{aligned}
\operatorname{HPFEOWG}\left(\bar{h}_{1}, \bar{h}_{2}\right) & =\left(\bar{h}_{\sigma(1)}^{\wedge_{\varepsilon} \omega_{1}}\right) \otimes_{\mathcal{E}}\left(\bar{h}_{\sigma(2)}^{\wedge_{\varepsilon} \omega_{2}}\right) \\
& =\{0.2937|0.1,0.2859| 0.1,0.4005|0.15,0.4466| 0.15,0.4530|0.25,0.5033| 0.25\}
\end{aligned}
$$

In the following section, we look at the HPFEOWA and HPFEOWG operators for some special cases of the associated vector $\omega$.

(1) If $\omega=(1,0, \ldots, 0)^{T}$, then

$$
\begin{aligned}
& \operatorname{HPFEOWA}\left(\bar{h}_{1}, \bar{h}_{2}, \ldots, \bar{h}_{T}\right)=\bar{h}_{\sigma(1)}=\max \left\{\bar{h}_{i}\right\}, \\
& \operatorname{HPFEOWG}\left(\bar{h}_{1}, \bar{h}_{2}, \ldots, \bar{h}_{n}\right)=\bar{h}_{\sigma(1)}=\max \left\{\bar{h}_{t}\right\} .
\end{aligned}
$$

(2) If $\omega=(0,0, \ldots, 1)^{T}$, then

$$
\begin{aligned}
& \operatorname{HPFEOWA}\left(\bar{h}_{1}, \bar{h}_{2}, \ldots, \bar{h}_{T}\right)=\bar{h}_{\sigma(T)}=\min \left\{\bar{h}_{t}\right\}, \\
& \operatorname{HPFEOWG}\left(\bar{h}_{1}, \bar{h}_{2}, \ldots, \bar{h}_{T}\right)=\bar{h}_{\sigma(T)}=\min \left\{\bar{h}_{t}\right\} .
\end{aligned}
$$

(3) If $\omega_{s}=1, w_{t}=0, s \neq t$, then

$$
\begin{aligned}
& \bar{h}_{\sigma(T)} \leq \operatorname{HPFEOWA}\left(\bar{h}_{1}, \bar{h}_{2}, \ldots, \bar{h}_{T}\right)=\bar{h}_{\sigma(s)} \leq \bar{h}_{\sigma(1)}, \\
& \bar{h}_{\sigma(T)} \leq \operatorname{HPFEOWG}\left(\bar{h}_{1}, \bar{h}_{2}, \ldots, \bar{h}_{T}\right)=\bar{h}_{\sigma(s)} \leq \bar{h}_{\sigma(1)},
\end{aligned}
$$

where $\bar{h}_{\sigma(s)}$ is the $s$ th largest $\bar{h}_{t}(t=1,2, \ldots, T)$. 
(4) If $\omega=\left(\frac{1}{T}, \frac{1}{T}, \ldots, \frac{1}{T}\right)^{T}$, then

$$
\begin{aligned}
& \operatorname{HPFEOWA}\left(\bar{h}_{1}, \bar{h}_{2}, \ldots, \bar{h}_{T}\right) \\
& =\bigcup_{\gamma_{\sigma(1)} \in \bar{h}_{\sigma(1)}, \gamma_{\sigma(2)} \in \bar{h}_{\sigma(2)} \ldots, \gamma_{\sigma(T)} \in \bar{h}_{\sigma(T)}}\left\{\frac{\prod_{t=1}^{T}\left(1+\gamma_{\sigma(t)}\right)^{\frac{1}{T}}-\prod_{t=1}^{T}\left(1-\gamma_{\sigma(t)}\right)^{\frac{1}{T}}}{\prod_{t=1}^{T}\left(1+\gamma_{\sigma(t)}\right)^{\frac{1}{T}}+\prod_{t=1}^{T}\left(1-\gamma_{\sigma(t)}\right)^{\frac{1}{T}}} \mid p_{\sigma(1)} p_{\sigma(2)} \cdots p_{\sigma(T)}\right\} \\
& =\bigcup_{\gamma_{1} \in \bar{h}_{1}, \gamma_{2} \in \bar{h}_{2}, \ldots, \gamma_{T} \in \bar{h}_{T}}\left\{\frac{\prod_{t=1}^{T}\left(1+\gamma_{t}\right)^{\frac{1}{T}}-\prod_{t=1}^{T}\left(1-\gamma_{t}\right)^{\frac{1}{T}}}{\prod_{t=1}^{T}\left(1+\gamma_{t}\right)^{\frac{1}{T}}+\prod_{t=1}^{T}\left(1-\gamma_{t}\right)^{\frac{1}{T}}} \mid p_{1} p_{2} \cdots p_{T}\right\} \\
& =\operatorname{HPFEA}\left(\bar{h}_{1}, \bar{h}_{2}, \ldots, \bar{h}_{T}\right) \text {, } \\
& \operatorname{HPFEOWA}\left(\bar{h}_{1}, \bar{h}_{2}, \ldots, \bar{h}_{T}\right) \\
& =\bigcup_{\gamma_{\sigma(1)} \in \bar{h}_{\sigma(1)}, \gamma_{\sigma(2)} \in \bar{h}_{\sigma(2)}, \ldots, \gamma_{\sigma(T)} \in \bar{h}_{\sigma(T)}}\left\{\frac{2 \prod_{t=1}^{T} \gamma_{\sigma(t)}^{\frac{1}{T}}}{\prod_{t=1}^{T}\left(2-\gamma_{\sigma(t)}\right)^{\frac{1}{T}}+\prod_{t=1}^{T} \gamma_{\sigma(t)}^{\frac{1}{T}}} \mid p_{\sigma(1)} p_{\sigma(2)} \cdots p_{\sigma(T)}\right\} \\
& =\bigcup_{\gamma_{1} \in \bar{h}_{1}, \gamma_{2} \in \bar{h}_{2}, \ldots, \gamma_{T} \in \bar{h}_{T}}\left\{\frac{2 \prod_{t=1}^{T} \gamma_{t}^{\frac{1}{T}}}{\prod_{t=1}^{T}\left(2-\gamma_{t}\right)^{\frac{1}{T}}+\prod_{t=1}^{T} \gamma_{t}^{\frac{1}{T}}} \mid p_{1} p_{2} \cdots p_{T}\right\} \\
& =\operatorname{HPFEG}\left(\bar{h}_{1}, \bar{h}_{2}, \ldots, \bar{h}_{T}\right) \text {, }
\end{aligned}
$$

i.e., the HPFEOWA (resp. HPFEOWG) operator is reduced to HPFEA (resp. HPFEG) operator.

Similar to Theorems 8 and 9, the above ordered weighted operators have the relationship below.

Theorem 10. If $\bar{h}_{t}(t=1,2, \ldots, T)$ is a collection of HPFEs, $\omega=\left(\omega_{1}, \omega_{2}, \ldots, \omega_{T}\right)^{T}$ is the associated vector of the aggregation operator such that $\omega_{t} \in[0,1]$ and $\sum_{t=1}^{T} \omega_{t}=1$. Then,

(1) $\operatorname{HPFEOWA}\left(\bar{h}_{1}, \bar{h}_{2}, \ldots, \bar{h}_{T}\right) \leq \operatorname{HPFOWA}\left(\bar{h}_{1}, \bar{h}_{2}, \ldots, \bar{h}_{T}\right)$;

(2) $\operatorname{HPFEOWG}\left(\bar{h}_{1}, \bar{h}_{2}, \ldots, \bar{h}_{T}\right) \geq \operatorname{HPFOWG}\left(\bar{h}_{1}, \bar{h}_{2}, \ldots, \bar{h}_{T}\right)$.

Theorem 11. If $\bar{h}_{t}(t=1,2, \ldots, T)$ is a collection of HPFEs, $\omega=\left(\omega_{1}, \omega_{2}, \ldots, \omega_{T}\right)^{T}$ is the associated vector of the aggregation operator, such that $\omega_{t} \in[0,1]$ and $\sum_{t=1}^{T} \omega_{t}=1$. Then,

(1) HPFEOWA $\left(\left(\bar{h}_{1}\right)^{c},\left(\bar{h}_{2}\right)^{c}, \ldots,\left(\bar{h}_{T}\right)^{c}\right)=\left(\operatorname{HPFEOWG}\left(\bar{h}_{1}, \bar{h}_{2}, \ldots, \bar{h}_{T}\right)\right)^{c}$;

(2) HPFEOWG $\left(\left(\bar{h}_{1}\right)^{c},\left(\bar{h}_{2}\right)^{c}, \ldots,\left(\bar{h}_{T}\right)^{c}\right)=\left(\operatorname{HPFEOWA}\left(\bar{h}_{1}, \bar{h}_{2}, \ldots, \bar{h}_{T}\right)\right)^{c}$.

Clearly, the fundamental characteristic of the HPFEWA and HPFEWG operators is that they consider the importance of each given HPFE, whereas the fundamental characteristic of the HPFEOWA and HPFEOWG operators is the weighting of the ordered positions of the HPFEs instead of weighting the given HPFEs themselves. By combining the advantages of the HPFEWA (resp. HPFEWG) and HPFEOWA (resp. HPFEOWG) operators, in the following text, we develop some hesitant probabilistic fuzzy hybrid aggregation operators that weight both the given HPFEs and their ordered positions.

Let $\bar{h}_{t}(t=1,2, \ldots, T)$ be a collection of HPFEs, $w=\left(w_{1}, w_{2}, \ldots, w_{T}\right)^{T}$ be the weight vector of $\bar{h}_{t}$ with $w_{t} \in[0,1]$ and $\sum_{t=1}^{T} w_{t}=1$, and $p_{t}$ be the probability of $\gamma_{t}$ in the HPFE $\bar{h}_{t}$. Then, we have the following two aggregation operators which are based on the mapping $H_{P}^{T} \rightarrow H_{P}$ with an associated vector $\omega=\left(\omega_{1}, \omega_{2}, \ldots, \omega_{T}\right)^{T}$, such that $\omega_{t} \in[0,1]$ and $\sum_{t=1}^{T} \omega_{t}=1$ :

(1) The hesitant probabilistic fuzzy Einstein hybrid averaging (HPFEHA) operator is

$$
\begin{aligned}
& \operatorname{HPFEHA}\left(\bar{h}_{1}, \bar{h}_{2}, \ldots, \bar{h}_{T}\right)=\left(\omega_{1} \cdot \dot{h}_{\sigma(1)}\right) \oplus_{\varepsilon}\left(\omega_{2} \cdot \dot{h}_{\sigma(2)}\right) \oplus_{\varepsilon} \cdots \oplus_{\varepsilon}\left(\omega_{T} \cdot \dot{h}_{\sigma(T)}\right)
\end{aligned}
$$

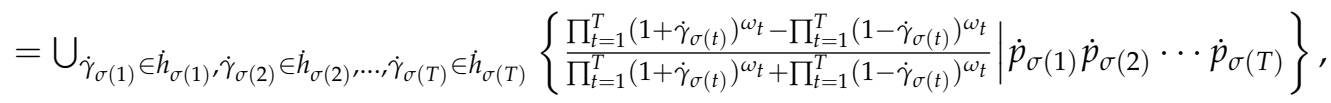


where $\dot{h}_{\sigma(t)}$ is the $t$ th largest of the weighted HPFEs $\dot{h}_{t}=T w_{t} \cdot{ }_{\varepsilon} \bar{h}_{t}(t=1,2, \ldots, T), T$ is the balancing coefficient, and $\dot{p}_{\sigma(t)}$ be the probability of $\dot{\gamma}_{\sigma(t)}$ in the HPFE $\dot{h}_{\sigma(t)}$.

(2) The hesitant probabilistic fuzzy Einstein hybrid geometric (HPFEHG) operator is

$$
\begin{aligned}
& \operatorname{HPFEHG}\left(\bar{h}_{1}, \bar{h}_{2}, \ldots, \bar{h}_{T}\right)=\left(\ddot{h}_{\sigma(1)}^{\wedge_{\varepsilon} \omega_{1}}\right) \otimes_{\mathcal{E}}\left(\ddot{h}_{\sigma(2)}^{\wedge_{\varepsilon} \omega_{2}}\right) \otimes_{\mathcal{E}} \cdots \otimes_{\mathcal{\varepsilon}}\left(\ddot{h}_{\sigma(T)}^{\wedge_{\varepsilon} \omega_{T}}\right)
\end{aligned}
$$

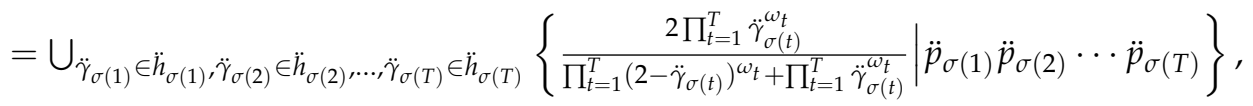

where $\ddot{h}_{\sigma(t)}$ is the $t$ th largest of the weighted HPFEs $\ddot{h}_{t}=\bar{h}_{t}^{\wedge_{\varepsilon} T w_{t}}(t=1,2, \ldots, T), T$ is the balancing coefficient, and $\ddot{p}_{\sigma(t)}$ is the probability of $\ddot{\gamma}_{\sigma(t)}$ in the HPFE $\ddot{h}_{\sigma(t)}$.

Especially, if $w=\left(\frac{1}{T}, \frac{1}{T}, \ldots, \frac{1}{T}\right)^{T}$, then $\dot{h}_{t}=\ddot{h}_{t}=\bar{h}_{t}(t=1,2, \ldots, T)$. In this case, the HPFEHA (resp. HPFEHG) operator is reduced to the HPFEOWA (resp. HPFEOWG) operator. If $\omega=\left(\frac{1}{T}, \frac{1}{T}, \ldots, \frac{1}{T}\right)^{T}$, then since $\frac{1}{T} \cdot \dot{h}_{t}=\frac{1}{T} \cdot \varepsilon\left(T w_{t} \cdot \varepsilon \bar{h}_{t}\right)=\cup_{\gamma_{t} \in \bar{h}_{t}}\left\{\frac{\left(1+\gamma_{t}\right)^{w_{t}}-\left(1-\gamma_{t}\right)^{w_{t}}}{\left(1+\gamma_{t}\right)^{w_{t}}+\left(1-\gamma_{t}\right)^{w_{t}}} \mid p_{t}\right\}$ and $\ddot{h}_{t}^{\wedge \varepsilon \frac{1}{T}}=\left(\bar{h}_{t}^{\wedge_{\varepsilon} T w_{t}}\right)^{\wedge_{\varepsilon} \frac{1}{T}}=\cup_{\gamma_{t} \in \bar{h}_{t}}\left\{\frac{2 \gamma_{t}^{w_{t}}}{\left(2-\gamma_{t}\right)^{w_{t}}+\gamma_{t}^{w_{t}}} \mid p_{t}\right\}$, we have

$$
\begin{aligned}
\operatorname{HPFEHA}\left(\bar{h}_{1}, \bar{h}_{2}, \ldots, \bar{h}_{T}\right) & =\left(\frac{1}{T} \cdot \dot{h}_{\sigma(1)}\right) \oplus_{\varepsilon}\left(\frac{1}{T} \cdot \dot{h}_{\varepsilon} \dot{h}_{\sigma(2)}\right) \oplus_{\varepsilon} \cdots \oplus_{\varepsilon}\left(\frac{1}{T} \cdot \dot{h}_{\sigma(T)}\right) \\
& =\bigcup_{\gamma_{1} \in \bar{h}_{1}, \gamma_{2} \in \bar{h}_{2}, \ldots, \gamma_{T} \in \bar{h}_{T}}\left\{\frac{\prod_{t=1}^{T}\left(1+\gamma_{t}\right)^{w_{t}}-\prod_{t=1}^{T}\left(1-\gamma_{t}\right)^{w_{t}}}{\prod_{t=1}^{T}\left(1+\gamma_{t}\right)^{w_{t}}+\prod_{t=1}^{T}\left(1-\gamma_{t}\right)^{w_{t}}} p_{1} p_{2} \cdots p_{T}\right\} \\
& =\operatorname{HPFEWA}\left(\bar{h}_{1}, \bar{h}_{2}, \ldots, \bar{h}_{T}\right),
\end{aligned}
$$

$$
\begin{aligned}
\operatorname{HPFEHG}\left(\bar{h}_{1}, \bar{h}_{2}, \ldots, \bar{h}_{T}\right) & =\left(\ddot{h}_{\sigma(1)}^{\wedge_{\varepsilon} \frac{1}{T}}\right) \otimes_{\mathcal{E}}\left(\ddot{h}_{\sigma(2)}^{\wedge_{\varepsilon} \frac{1}{T}}\right) \otimes_{\mathcal{E}} \cdots \otimes_{\mathcal{E}}\left(\ddot{h}_{\sigma(T)}^{\wedge_{\varepsilon} \frac{1}{T}}\right) \\
& =\bigcup_{\gamma_{1} \in \bar{h}_{1}, \gamma_{2} \in \bar{h}_{2}, \ldots, \gamma_{T} \in \bar{h}_{T}}\left\{\frac{2 \prod_{t=1}^{T} \gamma_{t}^{w_{t}}}{\prod_{t=1}^{T}\left(2-\gamma_{t}\right)^{w_{t}}+\prod_{t=1}^{T} \gamma_{t}^{w_{t}}} \mid p_{1} p_{2} \cdots p_{T}\right\} \\
& =\operatorname{HPFEWG}\left(\bar{h}_{1}, \bar{h}_{2}, \ldots, \bar{h}_{T}\right),
\end{aligned}
$$

i.e., the HPFEHA (resp. HPFEHG) operator is reduced to the HPFEWA (resp. HPFEWG) operator.

Example 6. Let $\bar{h}_{1}=(0.5|0.5,0.6| 0.5)$ and $\bar{h}_{2}=(0.1|0.2,0.3| 0.3,0.5 \mid 0.5)$ be two HPFEs. Suppose that the weight vector of them is $w=(0.63,0.37)^{T}$, and the aggregation associated vector is $\omega=(0.3,0.7)^{T}$. Then,

$$
\begin{aligned}
\dot{h}_{1}= & \left(\frac{(1+0.5)^{2 \times 0.63}-(1-0.5)^{2 \times 0.63}}{(1+0.5)^{2 \times 0.63}+(1-0.5)^{2 \times 0.63}}\left|0.5, \frac{(1+0.6)^{2 \times 0.63}-(1-0.6)^{2 \times 0.63}}{(1+0.6)^{2 \times 0.63}+(1-0.6)^{2 \times 0.63}}\right| 0.5\right) \\
= & (0.5993|0.5,0.7031| 0.5), \\
\dot{h}_{2}= & \left(\frac{(1+0.1)^{2 \times 0.37}-(1-0.1)^{2 \times 0.37}}{(1+0.1)^{2 \times 0.37}+(1-0.1)^{2 \times 0.37}}\left|0.2, \frac{(1+0.3)^{2 \times 0.37}-(1-0.3)^{2 \times 0.37}}{(1+0.3)^{2 \times 0.37}+(1-0.3)^{2 \times 0.37}}\right| 0.3,\right. \\
& \left.\frac{(1+0.5)^{2 \times 0.37}-(1-0.5)^{2 \times 0.37}}{(1+0.5)^{2 \times 0.37}+(1-0.5)^{2 \times 0.37}} \mid 0.2\right) \\
= & (0.7411|0.2,0.2251| 0.3,0.3851 \mid 0.5)
\end{aligned}
$$

and $s\left(\dot{h}_{1}\right)=0.6512$ and $s\left(\dot{h}_{2}\right)=0.4083$. Since $s\left(\dot{h}_{1}\right)>s\left(\dot{h}_{2}\right)$, we have

$$
\dot{h}_{\sigma(1)}=\dot{h}_{1}=(0.5993|0.5,0.7031| 0.5), \dot{h}_{\sigma(2)}=\dot{h}_{2}=(0.7411|0.2,0.2251| 0.3,0.3851 \mid 0.5) .
$$


From Equation (23), we have

$$
\begin{aligned}
\operatorname{HPFEHA}\left(\bar{h}_{1}, \bar{h}_{2}\right)= & \left(\omega_{1} \cdot \dot{h}_{\varepsilon(1)}\right) \oplus_{\mathcal{E}}\left(\omega_{2} \cdot \dot{h}_{\sigma(2)}\right) \\
= & \bigcup_{\dot{\gamma}_{\sigma(1)} \in \dot{h}_{\sigma(1)}, \dot{\gamma}_{\sigma(2)} \in \dot{h}_{\sigma(2)}}\left\{\frac{\prod_{t=1}^{2}\left(1+\dot{\gamma}_{\sigma(t)}\right)^{\omega_{t}}-\prod_{t=1}^{2}\left(1-\dot{\gamma}_{\sigma(t)}\right)^{\omega_{t}}}{\prod_{t=1}^{2}\left(1+\dot{\gamma}_{\sigma(t)}\right)^{\omega_{t}}+\prod_{t=1}^{2}\left(1-\dot{\gamma}_{\sigma(t)}\right)^{\omega_{t}}} \mid \dot{p}_{\sigma(1)} \dot{p}_{\sigma(2)}\right\} \\
& =\{0.3715|0.15,0.4175| 0.15,0.4557|0.25,0.4977| 0.25,0.7037|0.1,0.7302| 0.1\} .
\end{aligned}
$$

On the other hand,

$$
\begin{aligned}
\ddot{h}_{1} & =\left(\frac{2 \times 0.5^{2 \times 0.63}}{(2-0.5)^{2 \times 0.63}+0.5^{2 \times 0.63}}\left|0.5, \frac{2 \times 0.6^{2 \times 0.63}}{(2-0.6)^{2 \times 0.63}+0.6^{2 \times 0.63}}\right| 0.5\right) \\
& =(0.4007|0.5,0.5117| 0.5), \\
\ddot{h}_{2} & =\left(\frac{2 \times 0.1^{2 \times 0.37}}{(2-0.1)^{2 \times 0.37}+0.1^{2 \times 0.37}}\left|0.2, \frac{2 \times 0.3^{2 \times 0.37}}{(2-0.3)^{2 \times 0.37}+0.3^{2 \times 0.37}}\right| 0.3, \frac{2 \times 0.5^{2 \times 0.37}}{(2-0.5)^{2 \times 0.37}+0.5^{2 \times 0.37}} \mid 0.5\right) \\
& =(0.2033|0.2,0.4339| 0.3,0.6145 \mid 0.5)
\end{aligned}
$$

and since $s\left(\ddot{h}_{1}\right)=0.4562>0.4465=s\left(\ddot{h}_{2}\right)$, we have $\ddot{h}_{\sigma(1)}=\ddot{h}_{1}$ and $\ddot{h}_{\sigma(2)}=\ddot{h}_{2}$. From Equation (24), we have

$$
\begin{aligned}
& \operatorname{HPFEHG}\left(\bar{h}_{1}, \bar{h}_{2}\right)=\left(\ddot{h}_{\sigma(1)}^{\wedge_{\varepsilon} \omega_{1}}\right) \otimes_{\mathcal{\varepsilon}}\left(\ddot{h}_{\sigma(2)}^{\wedge_{\varepsilon} \omega_{2}}\right) \\
& =\bigcup_{\ddot{\gamma}_{\sigma(1)} \in \ddot{h}_{\sigma(1)}, \ddot{\gamma}_{\sigma(2)} \in \ddot{h}_{\sigma(2)}}\left\{\frac{2 \prod_{t=1}^{2} \gamma_{\sigma(t)}^{\omega_{t}}}{\prod_{t=1}^{2}\left(2-\gamma_{\sigma(t)}\right) \omega_{t}+\prod_{t=1}^{2} \gamma_{\sigma(t)}^{\omega_{t}}} \ddot{p}_{\sigma(1)} \ddot{p}_{\sigma(2)}\right\} \\
& =\{0.2512|0.1,0.2728| 0.1,0.4237|0.15,0.4563| 0.25,0.5441|0.15,0.5825| 0.25\} \text {. }
\end{aligned}
$$

\section{An Approach to MADM with Hesitant Probabilistic Fuzzy Information}

In this section, we utilize the proposed aggregation operators to develop an approach for MADM with hesitant probabilistic fuzzy information.

Let $X=\left\{x_{1}, x_{2}, \ldots, x_{n}\right\}$ be a set of $n$ alternatives and $G=\left\{g_{1}, g_{2}, \ldots, g_{m}\right\}$ be a set of $m$ attributes whose weight vector is $w=\left(w_{1}, w_{2}, \ldots, w_{m}\right)^{T}$, satisfying $w_{i}>0(i=1,2, \ldots, m)$ and $\sum_{i=1}^{m} w_{i}=1$, where $w_{i}$ denotes the importance degree of attribute $g_{i}$. Suppose the decision makers provide the evaluating values that the alternatives $x_{j}(i=1,2, \ldots, n)$ satisfy the attributes $g_{i}(j=1,2, \ldots, m)$ represented by the HPFEs $\bar{h}_{i j}\left(\gamma_{i j} \mid p_{i j}\right)(i=1,2, \ldots, m ; j=1,2, \ldots, n)$. All of these HPFEs are contained in the hesitant probabilistic fuzzy decision matrix $D=\left(\bar{h}_{i j}\left(\gamma_{i j} \mid p_{i j}\right)\right)_{m \times n}$ (see Table 1$)$.

Table 1. Hesitant probabilistic fuzzy decision matrix $(D)$.

\begin{tabular}{ccccc}
\hline & $\mathbf{x}_{\mathbf{1}}$ & $\mathbf{x}_{\mathbf{2}}$ & $\cdots$ & $\mathbf{x}_{\mathbf{n}}$ \\
\hline$g_{1}$ & $\bar{h}_{11}\left(\gamma_{11} \mid p_{11}\right)$ & $\bar{h}_{12}\left(\gamma_{12} \mid p_{12}\right)$ & $\cdots$ & $\bar{h}_{1 n}\left(\gamma_{1 n} \mid p_{1 n}\right)$ \\
$g_{2}$ & $\bar{h}_{21}\left(\gamma_{21} \mid p_{21}\right)$ & $\bar{h}_{22}\left(\gamma_{22} \mid p_{22}\right)$ & $\cdots$ & $\bar{h}_{2 n}\left(\gamma_{11} \mid p_{2 n}\right)$ \\
$\vdots$ & $\vdots$ & $\vdots$ & $\ddots$ & $\vdots$ \\
$g_{m}$ & $\bar{h}_{m 1}\left(\gamma_{m 1} \mid p_{m 1}\right)$ & $\bar{h}_{m 2}\left(\gamma_{m 2} \mid p_{m 2}\right)$ & $\cdots$ & $\bar{h}_{m n}\left(\gamma_{m n} \mid p_{m n}\right)$ \\
\hline
\end{tabular}

The following steps can be used to solve the MADM problem under the hesitant probabilistic fuzzy environment and obtain an optimal alternative.

Step 1: Obtain the normalized hesitant probabilistic fuzzy decision matrix. In general, the attribute set $(G)$ can be divided two subsets, $G_{1}$ and $G_{2}$, where $G_{1}$ and $G_{2}$ are the set of benefit attributes and cost attributes, respectively. If all of the attributes are of the same type, then the evaluation values do not need normalization, whereas if there are benefit attributes and cost attributes in MADM, in such 
cases, we may transform the evaluation values of cost type into the evaluation values of the benefit type by the following normalization formula:

$$
\bar{r}_{i j}\left(\beta_{i j} \mid p_{i j}\right)= \begin{cases}\bar{h}_{i j}, & i \in G_{1} \\ \bar{h}_{i j}^{c} & i \in G_{2}\end{cases}
$$

where $\bar{h}_{i j}^{c}=\cup_{\gamma_{i j} \in \bar{h}_{i j}}\left\{\left(1-\gamma_{i j}\right) \mid p_{i j}\right\}$ is the complement of $\bar{h}_{i j}$. Then, we obtain the normalized hesitant probabilistic fuzzy decision matrix $H=\left(\bar{r}_{i j}\left(\beta_{i j} \mid p_{i j}\right)\right)_{m \times n}$ (see Table 2).

Table 2. Normalized hesitant probabilistic fuzzy decision matrix $(H)$.

\begin{tabular}{ccccc}
\hline & $\mathbf{x}_{\mathbf{1}}$ & $\mathbf{x}_{\mathbf{2}}$ & $\cdots$ & $\mathbf{x}_{\mathbf{n}}$ \\
\hline$g_{1}$ & $\bar{r}_{11}\left(\beta_{11} \mid p_{11}\right)$ & $\bar{r}_{12}\left(\beta_{12} \mid p_{12}\right)$ & $\cdots$ & $\bar{r}_{1 n}\left(\beta_{1 n} \mid p_{1 n}\right)$ \\
$g_{2}$ & $\bar{r}_{21}\left(\beta_{21} \mid p_{21}\right)$ & $\bar{r}_{22}\left(\beta_{22} \mid p_{22}\right)$ & $\cdots$ & $\bar{r}_{2 n}\left(\beta_{11} \mid p_{2 n}\right)$ \\
$\vdots$ & $\vdots$ & $\vdots$ & $\ddots$ & $\vdots$ \\
$g_{m}$ & $\bar{r}_{m 1}\left(\beta_{m 1} \mid p_{m 1}\right)$ & $\bar{r}_{m 2}\left(\beta_{m 2} \mid p_{m 2}\right)$ & $\cdots$ & $\bar{r}_{m n}\left(\beta_{m n} \mid p_{m n}\right)$ \\
\hline
\end{tabular}

Step 2: Compute the overall assessment of alternatives. Utilize the HPFEWA operator

$$
\begin{aligned}
\bar{r}_{j} & =\operatorname{HPFEWA}\left(\bar{r}_{1 j}, \bar{r}_{2 j}, \ldots, \bar{r}_{m j}\right) \\
& =\bigcup_{\beta_{1 j} \in \bar{r}_{1 j}, \beta_{2 j} \in \bar{r}_{2 j}, \ldots, \beta_{m j} \in \bar{r}_{m j}}\left\{\frac{\prod_{i=1}^{m}\left(1+\beta_{i j}\right)^{w_{i}}-\prod_{i=1}^{m}\left(1-\beta_{i j}\right)^{w_{i}}}{\prod_{i=1}^{m}\left(1+\beta_{i j}\right)^{w_{i}}+\prod_{i=1}^{m}\left(1-\beta_{i j}\right)^{w_{i}}} \mid p_{1 j} p_{2 j} \cdots p_{m j}\right\}
\end{aligned}
$$

or the HPFEWG operator

$$
\begin{aligned}
\bar{r}_{j} & =\operatorname{HPFEWG}\left(\bar{r}_{1 j}, \bar{r}_{2 j}, \ldots, \bar{r}_{m j}\right) \\
& =\bigcup_{\beta_{1 j} \in \bar{r}_{1 j}, \beta_{2 j} \in \bar{r}_{2 j}, \ldots, \beta_{m j} \in \bar{r}_{m j}}\left\{\frac{2 \prod_{i=1}^{m}\left(\beta_{i j}\right)^{w_{i}}}{\prod_{i=1}^{m}\left(2-\beta_{i j}\right)^{w_{i}}+\prod_{i=1}^{m}\left(\beta_{i j}\right)^{w_{i}}} \mid p_{1 j} p_{2 j} \cdots p_{m j}\right\}
\end{aligned}
$$

to aggregate all the evaluating values $\bar{r}_{i j}(1=1,2, \ldots, m)$ of the $j$ th column and get the overall rating value $\bar{r}_{j}$ corresponding to the alternative $\left(x_{j}(j=1,2, \ldots, n)\right)$.

Step 3: Rank the order of all alternatives. Utilize the method in Definition 3 to rank the overall rating values $\bar{r}_{j}(j=1,2, \ldots, n)$. Rank all the alternatives $\left(x_{j}(j=1,2, \ldots, n)\right)$ in accordance with $\bar{r}_{j}$ $(j=1,2, \ldots, n)$ in descending order, and finally, select the most desirable alternative(s) with the largest overall evaluation value(s).

Step 4: End.

In the above-mentioned procedure, the HPFEWA (or HPFEWG) operator is utilized to aggregate the evaluating values of each alternative with respect to a collection of the attributes to rank and select the alternative(s). So we give a detail illustration of the decision making procedure with a propulsion/manoeuvring system selection problem.

Example 7. The propulsion/manoeuvring system selection is based on a study that was conducted for the selection of propulsion/manoeuvring system of a double ended passenger ferry to operate across the Bosphorus in Istanbul with the aim of reducing the journey time in highly congested seaway traffic (adopted from Ölçer and Odabaşi [43] and Wang and Liu [37]).

The propulsion/manoeuvring system alternatives are given as the set of alternatives $X=$ $\left\{x_{1}, x_{2}, x_{3}\right\}$. (1) $x_{1}$ is the conventional propeller and high lift rudder; (2) $x_{2}$ is the $Z$ drive; and (3) $x_{3}$ is the cycloidal propeller. The selection decision is made on the basis of one objective and seven subjective attributes, which are the following: (1) $g_{1}$ is the investment cost; (2) $g_{2}$ is the operating cost; 
(3) $g_{3}$ is the manoeuvrability; (4) $g_{4}$ is the propulsive power requirement; (5) $g_{5}$ is the reliability.; (6) $g_{6}$ is the propulsive power requirement; and (7) $g_{7}$ is the propulsive arrangement requirement. Note that the attributes are cost attributes, except for attributes $g_{3}$ and $g_{5}$, and the corresponding weight vector is $w=(0.15,0.2,0.3,0.2,0.15)^{T}$.

Assume that the decision makers use the linguistic terms shown in Table 3 to represent the evaluating values of the alternatives with respect to different attributes, respectively, and they provide their linguistic decision matrices $(D)$ as listed in Tables 4 .

Table 3. Linguistic terms and their corresponding hesitant probabilistic fuzzy elements (HPFEs).

\begin{tabular}{cc}
\hline Linguistic Terms & HPFEs \\
\hline Very low (VL) & $(0|0.7,0.1| 0.3)$ \\
Low (L) & $(0.15|0.6,0.25| 0.4)$ \\
Medium low (ML) & $(0.3|0.6,0.4| 0.4)$ \\
Medium (M) & $(0.45|0.5,0.55| 0.5)$ \\
Medium high (MH) & $(0.6|0.45,0.7| 0.55)$ \\
High (H) & $(0.75|0.4,0.85| 0.6)$ \\
Very high (VH) & $(0.9|0.4,1| 0.6)$ \\
\hline
\end{tabular}

Table 4. Linguistic decision matrix (D).

\begin{tabular}{lccc}
\hline & $\mathbf{x}_{\mathbf{1}}$ & $\mathbf{x}_{\mathbf{2}}$ & $\mathbf{x}_{\mathbf{3}}$ \\
\hline$g_{1}$ & $\mathrm{ML}$ & $\mathrm{M}$ & $\mathrm{H}$ \\
$g_{2}$ & $\mathrm{M}$ & $\mathrm{ML}$ & $\mathrm{H}$ \\
$g_{3}$ & $\mathrm{MH}$ & $\mathrm{M}$ & $\mathrm{MH}$ \\
$g_{4}$ & $\mathrm{H}$ & $\mathrm{H}$ & $\mathrm{L}$ \\
$g_{5}$ & $\mathrm{MH}$ & $\mathrm{MH}$ & $\mathrm{M}$ \\
$g_{6}$ & $\mathrm{H}$ & $\mathrm{M}$ & $\mathrm{M}$ \\
$g_{7}$ & $\mathrm{~L}$ & $\mathrm{MH}$ & $\mathrm{MH}$ \\
\hline
\end{tabular}

Step 1: Based on Tables 3 and 4, we can get the hesitant probabilistic fuzzy decision matrix $D=\left(\bar{h}_{i j}\right)_{7 \times 3}$ (see Table 5).

Table 5. Hesitant probabilistic fuzzy decision matrix $(D)$.

\begin{tabular}{cccc}
\hline & $\mathbf{x}_{\mathbf{1}}$ & $\mathbf{x}_{\mathbf{2}}$ & $\mathbf{x}_{\mathbf{3}}$ \\
\hline$g_{1}$ & $(0.3|0.6,0.4| 0.4)$ & $(0.45|0.5,0.55| 0.5)$ & $(0.75|0.4,0.85| 0.6)$ \\
$g_{2}$ & $(0.45|0.5,0.55| 0.5)$ & $(0.3|0.6,0.4| 0.4)$ & $(0.75|0.4,0.85| 0.6)$ \\
$g_{3}$ & $(0.6|0.45,0.7| 0.55)$ & $(0.45|0.5,0.55| 0.5)$ & $(0.6|0.45,0.7| 0.55)$ \\
$g_{4}$ & $(0.75|0.4,0.85| 0.6)$ & $(0.75|0.4,0.85| 0.6)$ & $(0.15|0.6,0.25| 0.4)$ \\
$g_{5}$ & $(0.6|0.45,0.7| 0.55)$ & $(0.6|0.45,0.7| 0.55)$ & $(0.45|0.5,0.55| 0.5)$ \\
$g_{6}$ & $(0.75|0.4,0.85| 0.6)$ & $(0.45|0.5,0.55| 0.5)$ & $(0.45|0.5,0.55| 0.5)$ \\
$g_{7}$ & $(0.15|0.6,0.25| 0.4)$ & $(0.6|0.45,0.7| 0.55)$ & $(0.6|0.45,0.7| 0.55)$ \\
\hline
\end{tabular}

Then, considering that the attributes are cost attributes, except for attributes $g_{3}$ and $g_{5}$, based on Equation (25), the hesitant probabilistic fuzzy decision matrix $(D)$ can be transformed into the following normalized hesitant probabilistic fuzzy decision matrix: $H=\left(\bar{r}_{i j}\right)_{7 \times 3}$ (see Table 6). 
Table 6. Normalized hesitant probabilistic fuzzy decision matrix $(H)$.

\begin{tabular}{cccc}
\hline & $\mathbf{x}_{\mathbf{1}}$ & $\mathbf{x}_{\mathbf{2}}$ & $\mathbf{x}_{\mathbf{3}}$ \\
\hline$g_{1}$ & $(0.6|0.4,0.7| 0.6)$ & $(0.45|0.5,0.55| 0.5)$ & $(0.15|0.6,0.25| 0.4)$ \\
$g_{2}$ & $(0.45|0.5,0.55| 0.5)$ & $(0.6|0.4,0.7| 0.6)$ & $(0.15|0.6,0.25| 0.4)$ \\
$g_{3}$ & $(0.6|0.45,0.7| 0.55)$ & $(0.45|0.5,0.55| 0.5)$ & $(0.6|0.45,0.7| 0.55)$ \\
$g_{4}$ & $(0.15|0.6,0.25| 0.4)$ & $(0.15|0.6,0.25| 0.4)$ & $(0.75|0.4,0.85| 0.6)$ \\
$g_{5}$ & $(0.6|0.45,0.7| 0.55)$ & $(0.6|0.45,0.7| 0.55)$ & $(0.45|0.5,0.55| 0.5)$ \\
$g_{6}$ & $(0.15|0.6,0.25| 0.4)$ & $(0.45|0.5,0.55| 0.5)$ & $(0.45|0.5,0.55| 0.5)$ \\
$g_{7}$ & $(0.75|0.4,0.85| 0.6)$ & $(0.3|0.55,0.4| 0.45)$ & $(0.3|0.55,0.4| 0.45)$ \\
\hline
\end{tabular}

Step 2: Utilize the decision information given in matrix $H$ and the HPFEWA operator (26) to derive the overall rating values $\left(\bar{r}_{j}\right)$ of the alternative $x_{j}(j=1,2,3)$ :

$$
\begin{aligned}
\bar{r}_{1}=\{ & 0.4953|0.0243,0.5148| 0.0297,0.5109|0.0162,0.5299| 0.0198,0.5337|0.0297,0.5521| 0.0363, \\
& 0.5484|0.0198,0.5664| 0.0242,0.5152|0.0243,0.5341| 0.0297,0.5304|0.0162,0.5489| 0.0198, \\
& 0.5525|0.0297,0.5704| 0.0363,0.5669|0.0198,0.5843| 0.0242,0.5148|0.0365,0.5337| 0.0446, \\
& 0.5299|0.0243,0.5484| 0.0297,0.5521|0.0446,0.5700| 0.0545,0.5664|0.0297,0.5839| 0.0363, \\
& 0.5341|0.0365,0.5525| 0.0446,0.5489|0.0243,0.5669| 0.0297,0.5704|0.0446,0.5878| 0.0545, \\
& 0.5843|0.0297,0.6013| 0.0363\},
\end{aligned}
$$

$$
\begin{aligned}
\bar{r}_{2}=\{ & 0.4550|0.0270,0.4754| 0.0330,0.4713|0.0180,0.4914| 0.0220,0.4862|0.0270,0.5059| 0.0330, \\
& 0.5019|0.0180,0.5212| 0.0220,0.4821|0.0405,0.5019| 0.0495,0.4980|0.0270,0.5174| 0.0330, \\
& 0.5123|0.0405,0.5313| 0.0495,0.5275|0.0270,0.5461| 0.0330,0.4707|0.0270,0.4908| 0.0330, \\
& 0.4868|0.0180,0.5065| 0.0220,0.5013|0.0270,0.5206| 0.0330,0.5168|0.0180,0.5357| 0.0220, \\
& 0.4974|0.0405,0.5168| 0.0495,0.5129|0.0270,0.5319| 0.0330,0.5270|0.0405,0.5456| 0.0495, \\
& 0.5419|0.0270,0.5601| 0.0330\},
\end{aligned}
$$

$\bar{r}_{3}=\{0.4840|0.0324,0.4992| 0.0324,0.5261|0.0486,0.5404| 0.0486,0.5230|0.0396,0.5374| 0.0396$, $0.5629|0.0594,0.5764| 0.0594,0.4997|0.0216,0.5146| 0.0216,0.5410|0.0324,0.5550| 0.0324$, $0.5379|0.0264,0.5520| 0.0264,0.5769|0.0396,0.5902| 0.0396,0.4958|0.0216,0.5108| 0.0216$, $0.5373|0.0324,0.5514| 0.0324,0.5342|0.0264,0.5484| 0.0264,0.5734|0.0396,0.5867| 0.0396$, $0.5114|0.0144,0.5260| 0.0144,0.5519|0.0216,0.5657| 0.0216,0.5489|0.0176,0.5628| 0.0176$, $0.5873|0.0264,0.6002| 0.0264\}$.

Step 3: Calculate the score values of the overall rating values $\left(\bar{r}_{j}\right)$ of the alternatives $\left(x_{j}(j=1,2,3)\right)$ :

$$
s\left(\bar{r}_{1}\right)=0.5533, s\left(\bar{r}_{2}\right)=0.5110, s\left(\bar{r}_{3}\right)=0.5473 .
$$

Since $s\left(\bar{r}_{1}\right)>s\left(\bar{r}_{3}\right)>s\left(\bar{r}_{2}\right)$, the ranking order of the alternatives $x_{j}(j=1,2,3)$ is

$$
x_{1} \succ x_{3} \succ x_{2} .
$$

Therefore, the best alternative is $x_{1}$. 
If we utilize the HPFEWG operator (27) in Step 2 to get the overall rating values $\left(\bar{r}_{j}\right)$ of the alternatives $\left(x_{j}(j=1,2,3)\right)$, we obtain

$$
\begin{aligned}
\bar{r}_{1}=\{ & 0.4426|0.0243,0.4545| 0.0297,0.4828|0.0162,0.4955| 0.0198,0.4666|0.0297,0.4790| 0.0363, \\
& 0.5083|0.0198,0.5215| 0.0242,0.4613|0.0243,0.4736| 0.0297,0.5027|0.0162,0.5127| 0.0198, \\
& 0.4860|0.0297,0.4987| 0.0363,0.5289|0.0198,0.5423| 0.0242,0.4545|0.0365,0.4667| 0.0446, \\
& 0.4955|0.0243,0.5084| 0.0297,0.4790|0.0446,0.4916| 0.0545,0.5215|0.0297,0.5348| 0.0363, \\
& 0.4726|0.0365,0.4861| 0.0446,0.5157|0.0243,0.5290| 0.0297,0.4987|0.0446,0.5117| 0.0545, \\
& 0.5423|0.0297,0.5560| 0.0363\},
\end{aligned}
$$

$$
\begin{aligned}
\bar{r}_{2}=\{ & 0.4100|0.0270,0.4213| 0.0330,0.4481|0.0180,0.4602| 0.0220,0.4367|0.0270,0.4485| 0.0330, \\
& 0.4766|0.0180,0.4892| 0.0220,0.4250|0.0405,0.4366| 0.0495,0.4641|0.0270,0.4765| 0.0330, \\
& 0.4525|0.0405,0.4646| 0.0495,0.4933|0.0270,0.5062| 0.0330,0.4232|0.0270,0.4347| 0.0330, \\
& 0.4622|0.0180,0.4745| 0.0220,0.4505|0.0270,0.4626| 0.0330,0.4913|0.0180,0.5041| 0.0220, \\
& 0.4386|0.0405,0.4505| 0.0495,0.4786|0.0270,0.4912| 0.0330,0.4666|0.0405,0.4790| 0.0495, \\
& 0.5084|0.0270,0.5215| 0.0330\},
\end{aligned}
$$

$$
\begin{aligned}
\bar{r}_{3}=\{ & 0.3890|0.0324,0.4017| 0.0324,0.4022|0.0486,0.4152| 0.0486,0.4109|0.0396,0.4241| 0.0396, \\
& 0.4246|0.0594,0.4382| 0.0594,0.4257|0.0216,0.4393| 0.0216,0.4398|0.0324,0.4537| 0.0324, \\
& 0.4491|0.0264,0.4632| 0.0264,0.4638|0.0396,0.4782| 0.0396,0.4163|0.0216,0.4297| 0.0216, \\
& 0.4302|0.0324,0.4439| 0.0324,0.4393|0.0264,0.4532| 0.0264,0.4538|0.0396,0.4680| 0.0396, \\
& 0.4549|0.0144,0.4691| 0.0144,0.4697|0.0216,0.4843| 0.0216,0.4794|0.0176,0.49421| 0.0176, \\
& 0.4948|0.0264,0.5098| 0.0264\} .
\end{aligned}
$$

Then, we calculate the scores of the overall rating values $\bar{r}_{j}$ of the alternatives:

$$
s\left(\bar{r}_{1}\right)=0.4968, s\left(\bar{r}_{2}\right)=0.4621, s\left(\bar{r}_{3}\right)=0.4429 .
$$

Since $s\left(\bar{r}_{1}\right)>s\left(\bar{r}_{2}\right)>s\left(\bar{r}_{3}\right)$, the ranking order of the alternatives $x_{j}(j=1,2,3)$ is

$$
x_{1} \succ x_{2} \succ x_{3} .
$$

Then, the best alternative is also $x_{1}$.

In order to compare the performance with the existing operators, in the following text, the HPFWA operator (2) and HPFWG operator (3) proposed by Xu and Zhou [27] are used to computing the overall rating values. If we first utilize the HPFWA operator (2) presented in Step 2, then we get the overall rating values $\bar{r}_{j}$ of the alternatives $\left(x_{j}(j=1,2,3)\right)$ :

$$
\begin{aligned}
\bar{r}_{1}=\{ & 0.5043|0.0243,0.5252| 0.0297,0.5165|0.0162,0.5369| 0.0198,0.5453|0.0297,0.5645| 0.0363, \\
& 0.5566|0.0198,0.5753| 0.0242,0.5238|0.0243,0.5439| 0.0297,0.5356|0.0162,0.5552| 0.0198, \\
& 0.5632|0.0297,0.5817| 0.0363,0.5740|0.0198,0.5920| 0.0242,0.5252|0.0365,0.5453| 0.0446, \\
& 0.5369|0.0243,0.5565| 0.0297,0.5645|0.0446,0.5829| 0.0545,0.5753|0.0297,0.5932| 0.0363, \\
& 0.5439|0.0365,0.5632| 0.0446,0.5552|0.0243,0.5739| 0.0297,0.5817|0.0446,0.5993| 0.0545, \\
& 0.5920|0.0297,0.6092| 0.0363\},
\end{aligned}
$$


$\bar{r}_{2}=\{0.4632|0.0270,0.4859| 0.0330,0.4765|0.0180,0.4986| 0.0220,0.4946|0.0270,0.5159| 0.0330$, $0.5071|0.0180,0.5279| 0.0220,0.4933|0.0405,0.5147| 0.0495,0.5058|0.0270,0.5267| 0.0330$, $0.5229|0.0405,0.5430| 0.0495,0.5347|0.0270,0.5543| 0.0330,0.4792|0.0270,0.5012| 0.0330$, $0.4920|0.0180,0.5135| 0.0220,0.5096|0.0270,0.5303| 0.0330,0.5217|0.0180,0.5419| 0.0220$, $0.5083|0.0405,0.5291| 0.0495,0.5205|0.0270,0.5407| 0.0330,0.5370|0.0405,0.5566| 0.0495$, $0.5485|0.0270,0.5675| 0.0330\}$,

$\bar{r}_{3}=\{0.5027|0.0324,0.5175| 0.0324,0.5510|0.0486,0.5643| 0.0486,0.5439|0.0396,0.5574| 0.0396$, $0.5882|0.0594,0.6004| 0.0594,0.5150|0.0216,0.5294| 0.0216,0.5621|0.0324,0.5751| 0.0324$, $0.5552|0.0264,0.5684| 0.0264,0.5984|0.0396,0.6103| 0.0396,0.5120|0.0216,0.5264| 0.0216$, $0.5594|0.0324,0.5724| 0.0324,0.5524|0.0264,0.5656| 0.0264,0.5958|0.0396,0.6078| 0.0396$, $0.5240|0.0144,0.5381| 0.0144,0.5702|0.0216,0.5810| 0.0216,0.5634|0.0176,0.5764| 0.0176$, $0.6058|0.0264,0.6175| 0.0264\}$.

Then, the scores of the overall rating values $\left(\bar{r}_{j}(j=1,2,3)\right)$ are $s\left(\bar{r}_{1}\right)=0.5630, s\left(\bar{r}_{2}\right)=0.5202$, and $s\left(\bar{r}_{3}\right)=0.5672$, and so, the ranking order of the alternatives $\left(x_{j}(j=1,2,3)\right)$ is $x_{3} \succ x_{1} \succ x_{2}$. Thus, the best alternative is $x_{3}$.

Next, if we utilize the HPFWG operator (3) presented in Step 2, we get the overall rating values $\left(\bar{r}_{j}\right)$ of the alternatives $x_{j}(j=1,2,3)$ :

$\bar{r}_{1}=\{0.4293|0.0243,0.4393| 0.0297,0.4754|0.0162,0.4866| 0.0198,0.4496|0.0297,0.4601| 0.0363$, $0.4979|0.0198,0.5096| 0.0242,0.4469|0.0243,0.4573| 0.0297,0.4949|0.0162,0.5065| 0.0198$, $0.4680|0.0297,0.4790| 0.0363,0.5183|0.0198,0.5305| 0.0242,0.4393|0.0365,0.4496| 0.0446$, $0.4866|0.0243,0.4980| 0.0297,0.4601|0.0446,0.4709| 0.0545,0.5096|0.0297,0.5216| 0.0363$, $0.4573|0.0365,0.4680| 0.0446,0.5065|0.0243,0.5184| 0.0297,0.4790|0.0446,0.4902| 0.0545$, $0.5305|0.0297,0.5429| 0.0363\}$,

$\bar{r}_{2}=\{0.3995|0.0270,0.4089| 0.0330,0.4425|0.0180,0.4529| 0.0220,0.4243|0.0270,0.4342| 0.0330$, $0.4699|0.0180,0.4809| 0.0220,0.4120|0.0405,0.4217| 0.0495,0.4563|0.0270,0.4670| 0.0330$, $0.4375|0.0405,0.4478| 0.0495,0.4846|0.0270,0.4960| 0.0330,0.4117|0.0270,0.4214| 0.0330$, $0.4560|0.0180,0.4667| 0.0220,0.4373|0.0270,0.4475| 0.0330,0.4843|0.0180,0.4956| 0.0220$, $0.4246|0.0405,0.4345| 0.0495,0.4703|0.0270,0.4813| 0.0330,0.4509|0.0405,0.4615| 0.0495$, $0.4994|0.0270,0.5111| 0.0330\}$,

$\bar{r}_{3}=\{0.3699|0.0324,0.3812| 0.0324,0.3792|0.0486,0.3908| 0.0486,0.3874|0.0396,0.3992| 0.0396$, $0.3972|0.0594,0.4093| 0.0594,0.4097|0.0216,0.4222| 0.0216,0.4200|0.0324,0.4329| 0.0324$, $0.4291|0.0264,0.4422| 0.0264,0.4399|0.0396,0.4534| 0.0396,0.3994|0.0216,0.4116| 0.0216$, $0.4095|0.0324,0.4220| 0.0324,0.4183|0.0264,0.4311| 0.0264,0.4289|0.0396,0.4420| 0.0396$, $0.4423|0.0144,0.4559| 0.0144,0.4535|0.0216,0.4674| 0.0216,0.4633|0.0176,0.4774| 0.0176$, $0.4750|0.0264,0.4895| 0.0264\}$. 
Then, the scores of the overall rating values $\left(\bar{r}_{j}(j=1,2,3)\right)$ are $s\left(\bar{r}_{1}\right)=0.4817, s\left(\bar{r}_{2}\right)=0.4501$, and $s\left(\bar{r}_{3}\right)=0.4210$, and so the ranking order of the alternatives $\left(x_{j}(j=1,2,3)\right)$ is $x_{1} \succ x_{2} \succ x_{3}$. Thus, the best alternative is $x_{1}$.

The relative comparison of the methods using different operators proposed by $\mathrm{Xu}$ and Zhou [27] is shown in Table 7. From Table 7, we can see that the obtained overall rating values of the alternatives are different with each of the four operators, respectively, and then, the ranking orders of the alternatives also are different. Each of the methods using different hesitant probabilistic fuzzy operators has its advantages and disadvantages, and none of them always perform better than the others in any situation. It depends on how we look at things, and not on how they are themselves.

Table 7. Comparison of overall rating values and ranking orders of alternatives.

\begin{tabular}{ccc}
\hline Aggregation Operator & Overall Rating Values & Ranking Orders \\
\hline HPFWA operator [27] & $s\left(\bar{r}_{1}\right)=0.5630, s\left(\bar{r}_{2}\right)=0.5202, s\left(\bar{r}_{3}\right)=0.5672$ & $x_{3} \succ x_{1} \succ x_{2}$ \\
HPFWG operator [27] & $s\left(\bar{r}_{1}\right)=0.4817, s\left(\bar{r}_{2}\right)=0.4501, s\left(\bar{r}_{3}\right)=0.4210$ & $x_{1} \succ x_{2} \succ x_{3}$ \\
HPFEWA operator & $s\left(\bar{r}_{1}\right)=0.5533, s\left(\bar{r}_{2}\right)=0.5110, s\left(\bar{r}_{3}\right)=0.5473$ & $x_{1} \succ x_{3} \succ x_{2}$ \\
HPFEWG operator & $s\left(\bar{r}_{1}\right)=0.4968, s\left(\bar{r}_{2}\right)=0.4621, s\left(\bar{r}_{3}\right)=0.4429$ & $x_{1} \succ x_{2} \succ x_{3}$ \\
\hline
\end{tabular}

Consequently, the use of different hesitant probabilistic fuzzy aggregation operators reflects the decision maker's pessimistic (or optimistic) attribute. For example, the proposed HPFEWA operator shows that the decision maker has a more pessimistic attribute than the HPFWA operator [27], and the proposed HPFEWG operator shows that the decision maker has a more optimistic attribute than the HPFWG operator [27] in the aggregation process.

\section{Conclusions}

The hesitant probabilistic fuzzy MADM is an important research topic in HPFS theory and decision science with uncertain information. Information aggregation is one of the core issues. Based on the Einstein operational rules of HPFEs, in this paper, we developed a series of hesitant probabilistic fuzzy Einstein aggregation operators, including the HPFEWA, HPFEWG, HPFEOWA, HPFEOWG, HPFEHA, and HPFEHG operators. Some basic properties of the proposed aggregation operators, such as boundedness and monotonicity, and the relationships between them were investigated. We compared the proposed operators with the existing hesitant probabilistic fuzzy aggregation operators proposed by $\mathrm{Xu}$ and Zhou [27] and presented corresponding relations. These proposed hesitant probabilistic Einstein aggregation operators provide a fine supplement to the existing work on HPFSs. Based on the HPFEWA and HPFEWG operators, a new method for MADM was developed in hesitant probabilistic fuzzy environments. A practical example was provided to illustrate the hesitant probabilistic fuzzy MADM process. Through a comparison between the proposed method with the previously proposed hesitant probabilistic fuzzy MADM method [27], we showed some advantages of the proposed hesitant probabilistic fuzzy MADM method.

This paper only considered decision makers with equl weights in the decision making process, but further studies on unequal weights are needed. Moreover, research using other operations, such as Hamacher and Frank $t$-conoms and $t$-norms instead of the Einstein t-conorm and t-norm, should be discussed in future studies.

Author Contributions: J.H.P. drafted the initial manuscript and conceived the MADM framework. M.J.S. provided the relevant literature review and illustrated example. J.H.P. and Y.K.P. revised the manuscript and analyzed the data.

Funding: This work was supported by a Research Grant from Pukyong National University (2017).

Conflicts of Interest: The authors declare no conflict of interest. 


\section{References}

1. Milosavljevića, M.; Bursaća, M.; Tričkovića, G. Selection of the railroad container terminal in Serbia based on multi criteria decision-making methods. Decis. Mak. Appl. Manag. Eng. 2018, 1. [CrossRef]

2. Badi, I.; Ballem, M. Supplier selection using the rough BWM-MAIRCA model: A case study in pharmaceutical supplying in Libya. Decis. Maki. Appl. Manag. Eng. 2018, 1. [CrossRef]

3. Jagannath Roy, J.; Krishnedu Adhikary, K.; Samarjit Kar, S.; Dragan Pamucar, D. A rough strength relational DEMATEL model for analysing the key success factors of hospital service quality. Decis. Mak. Appl. Manag. Eng. 2018, 1, 121-142.

4. Zadeh, L.A. Information and control. Fuzzy Sets 1965, 8, 338-353.

5. Zhou, W. An accurate method for determining hesitant fuzzy aggregation operator weights and its application to project investment. Int. J. Intell. Syst. 2014, 29, 668-686. [CrossRef]

6. Zhou, W.; Xu, Z.S. Generalized asymmetric linguistic term set and its application to qualitative decision making involving risk appetities. Eur. J. Oper. Res. 2016, 254, 610-621. [CrossRef]

7. Dong, Y.C.; Chen, X.; Herrera, F. Minimizing adjusted simple terms in the consensus reaching process with hesitant linguistic assessments in group decision making. Inf. Sci. 2015, 297, 95-117. [CrossRef]

8. Gou, X.J.; Xu, Z.S. Exponential operations for intuitionistic fuzzy numbers and interval numbers in multi-attribute decision making. Fuzzy Optim. Decis. Making 2016. [CrossRef]

9. Atanassov, K. Intuitionistic fuzzy sets. Fuzzy Sets Syst. 1986, 20, 87-96. [CrossRef]

10. Dubois, D.; Prade, H. Fuzzy Sets and Systems: Theory and Applications; Academic Press: Cambridge, MA, USA, 1980.

11. Miyamoto, S. Fuzzy multisets and their generalizations. In Proceedings of the International Conference on Membrane Computing, Curtea de Arges, Romania, 21-25 August 2000, pp. 225-236.

12. Torra, V. Hesitant fuzzy sets. Int. J. Intell. Syst. 2010, 25, 529-539. [CrossRef]

13. Xia, M.M.; Xu, Z.S. Hesitant fuzzy information aggregation in decision making. Int. J. Approx. Reason. 2011, 52, 395-407. [CrossRef]

14. Li, D.Q.; Zeng, W.Y.; Zhao, Y.B. Note on distance measure of hesitant fuzzy sets. Inf. Sci. 2015, 321, $103-115$. [CrossRef]

15. Meng, F.Y.; Chen, X.H. Correlation coefficients of hesitant fuzzy sets and their application based on fuzzy measures. Cognit. Comput. 2015, 7, 445-463. [CrossRef]

16. Bedregal, B.; Reiser, R.; Bustince, H.; Lopez-Molina, C.; Torra, V. Aggregation functions for typical hesitant fuzzy elements and the cation of automorphisms. Inf. Sci. 2014, 255, 82-99. [CrossRef]

17. Xu, Z.S.; Cai, X.Q. Recent advances in intuitionistic fuzzy information aggregation. Fuzzy Optim. Decis. Mak. 2010, 9, 359-381. [CrossRef]

18. Zhu, B.; Xu, Z.S.; Xia, M.M. Hesitant fuzzy geometric Bonferroni means. Inf. Sci. 2012, 205, 72-85. [CrossRef]

19. Xia, M.M.; Xu, Z.S.; Chen, N. Some hesitant fuzzy aggregation operators with their application in group decision making. Group Dec. Negoit. 2013, 22, 259-279. [CrossRef]

20. Zhang, Z.M. Hesitant fuzzy power aggregation operators and their application to multiple attribute group decision making. Inf. Sci. 2013, 234, 150-181. [CrossRef]

21. Yu, D.J. Some hesitant fuzzy infromation aggregation operators based on Einstein operational laws. Int. J. Intell. Syst. 2014, 29, 320-340. [CrossRef]

22. Zhou, X.; Li, Q. Multiple attribute decision making based on hesitant fuzzy Einstein geometric aggregation operators. J. App. Math. 2014, 2014. [CrossRef]

23. Zhou, X.; Li, Q. Generalized hesitant fuzzy prioritized Einstein aggregation operators and their application in group decision making. Int. J. Fuzzy. Syst. 2014, 16, 303-316.

24. Liao, H.C.; Xu, Z.S. A VIKOR-based method for hesitant fuzzy multi-criteria decision making. Fuzz. Optim. Decis. Mak. 2016, 12, 372-392. [CrossRef]

25. Rodriguez, R.M.; Martínez, L.; Torra, V.; Xu, Z.S.; Herrera, F. Hesitant fuzzy sets: State of the art and future directions. Int. J. Intell. Syst. 2014, 29, 495-524. [CrossRef]

26. Zhou, W.; Xu, Z.S. Asmmetric hesitant fuzzy sigmoid preference relations in analytic hierarchy process. Inf. Sci. 2016, 358, 191-207. [CrossRef]

27. Xu, Z.S.; Zhou, W. Consensus building with a group of decision makers under the hesitant probabilistic fuzzy environment. Fuzz. Optim. Decis. Mak. 2017, 16, 481-503. [CrossRef] 
28. Zhang, Z.; Wu, C. Weighted hesitant fuzzy sets and their application to multi-criteria decision making. Br. J. Math. Comput. Sci. 2014, 4, 1091-1123. [CrossRef]

29. Bedregal, B.; Beliakov, G.; Bustine, H.; Calvo, T.; Mesiar, R.; Patermain, D. A class of fuzzy multisets with a fixed number of memberships. Inf. Sci. 2012, 189, 1-17. [CrossRef]

30. Wang, R.; Li, Y.. Picture hesitant fuzzy set and its application to multiple criteria decision-making. Symmetry 2018, 10, 295-324. [CrossRef]

31. Chen, N.; Xu, Z.S.; Xia, M.M. Interval-valued hesitant preference relations and their applications to group decision making. Knowl. Based Syst. 2013, 37, 528-540. [CrossRef]

32. Pathinathan, T.; Savarimuthu, J.S. Trapezoidal hesitant fuzzy multi-attribute decision making based on TOPSIS. Int. Arch. App. Sci. Technol. 2015, 6, 39-49.

33. Yu, D. Triangular hesitant fuzzy set and its application to teaching quality evaluation. J. Inform. Comput. Sci. 2013, 10, 1925-1934. [CrossRef]

34. Mahmood, T.; Mehmood F.; Khan, Q. Cubic hesitant fuzzy sets and their applications to multi criteria decision making. Int. J. Algebra Stat. 2016, 5, 19-51. [CrossRef]

35. Klement, E.; Mesiar, R.; Pap, E. Book Review: "Triangular Norms". Int. J. Uncertain. Fuzz. Knowl. Based Syst. 2003, 11, 257-259. [CrossRef]

36. Wang, W.Z.; Liu, X.W. Intuitionistic fuzzy geometric aggregation operators based on Einstein operations. Int. J. Intell. Syst. 2011, 26, 1049-1075. [CrossRef]

37. Wang, W.Z.; Liu, X.W. Intuitionistic fuzzy infromation aggregation using Einstein operations. IEEE Trans. Fuzzy Syst. 2012, 20, 923-938. [CrossRef]

38. Zhao, X.; Wei, G. Some intuitionistic fuzzy Einstein hybrid aggregation operators and their application to multiple attribute decision making. Knowl. Based Syst. 2013, 37, 472-479. [CrossRef]

39. Yager, R.R. On ordered weighted averaging aggregation operators in multi-criteria decision making. IEEE Trans. Syst. Man Cybern. 1988, 18, 183-190. [CrossRef]

40. $\mathrm{Xu}, \mathrm{Z} . \mathrm{S}$. An overview of methods for determining OWA weights. Int. J. Intell. Syst. 2005, 20, 843-865. [CrossRef]

41. $\mathrm{Xu}, \mathrm{Z}$.S. On consistency of the weighted geometric mean complex judgement matrix in AHP. Eur. J. Oper. Res. 2000, 126, 683-687. [CrossRef]

42. Torra, V.; Narukawa, Y. Modeling Decisions: Information Fusion and Aggregation Operators; Springer: Berlin/Heidelberg, Germany, 2007.

43. Ölçer, A.I.; Odabaşi, A.Y. A new fuzzy multiple attribute group decision making methodology and its application to propulsion/manoeuvring system selectio problem. Eur. J. Oper. Res. 2005, 166, 93-114. [CrossRef]

(C) 2018 by the authors. Licensee MDPI, Basel, Switzerland. This article is an open access article distributed under the terms and conditions of the Creative Commons Attribution (CC BY) license (http://creativecommons.org/licenses/by/4.0/). 